\title{
Long noncoding RNA LERFS negatively regulates rheumatoid synovial aggression and proliferation
}

\author{
Yaoyao Zou, ${ }^{1}$ Siqi Xu, ${ }^{1}$ Youjun Xiao, ${ }^{1}$ Qian Qiu, ${ }^{1}$ Maohua Shi, ${ }^{1}$ Jingnan Wang, ${ }^{1}$ Liuqin Liang, ${ }^{1}$ Zhongping Zhan, ${ }^{1}$ Xiuyan Yang, \\ Nancy Olsen, ${ }^{2}$ Song Guo Zheng, ${ }^{2}$ and Hanshi $\mathrm{Xu}^{1}$ \\ 'Department of Rheumatology and Clinical Immunology, The First Affiliated Hospital, Sun Yat-sen University, Guangzhou, Guangdong, China. '2Department of Medicine, Milton S. Hershey Medical Center, \\ Pennsylvania State University, Hershey, Pennsylvania, USA.
}

\begin{abstract}
Fibroblast-like synoviocytes (FLSs) are critical to synovial aggression and joint destruction in rheumatoid arthritis (RA). The role of long noncoding RNAs (IncRNAs) in RA is largely unknown. Here, we identified a IncRNA, LERFS (lowly expressed in rheumatoid fibroblast-like synoviocytes), that negatively regulates the migration, invasion, and proliferation of FLSs through interaction with heterogeneous nuclear ribonucleoprotein $Q$ (hnRNP Q). Under healthy conditions, by binding to the mRNA of RhoA, Rac1, and CDC42 - the small CTPase proteins that control the motility and proliferation of FLSs - the LERFS-hnRNP $\mathrm{Q}$ complex decreased the stability or translation of target mRNAs and downregulated their protein levels. But in RA FLSs, decreased LERFS levels induced a reduction of the LERFS-hnRNP $Q$ complex, which reduced the binding of hnRNP $Q$ to target mRNA and therefore increased the stability or translation of target mRNA. These findings suggest that a decrease in synovial LERFS may contribute to synovial aggression and joint destruction in RA and that targeting the IncRNA LERFS may have therapeutic potential in patients with RA.
\end{abstract}

\section{Introduction}

Rheumatoid arthritis (RA) is a common autoimmune disease characterized by persistent joint inflammation and destruction of cartilage and bone (1). Fibroblast-like synoviocytes (FLSs), the unique cells in the synovial intimal lining, are a prominent component of hyperplastic synovial pannus tissue, which mediates cartilage and bone damage. RA FLSs display important immunomodulatory functions through the secretion of inflammatory mediators and direct interactions with synovia-infiltrating immune cells such as T cells and macrophages. However, RA FLSs also exhibit surprisingly aggressive behavior, including resistance to apoptosis, anchorage-independent growth, increased migration, and the ability to invade; this last trait is why RA FLSs are known as "transformed aggressors" or "imprinted aggressors" (2-5). Accumulating evidence suggests that regulating activated FLS aggression may be a new therapeutic strategy for reducing joint damage in RA. The invasive phenotype of RA FLSs depends on both an abnormal synovial microenvironment and autonomous factors that include somatic mutations or epigenetic changes (5-9). What causes FLSs to serve as imprinted aggressors in the progression of $\mathrm{RA}$ is as yet unknown.

Over the past decade, it has been shown that up to $90 \%$ of the human genome is transcribed, but only $1.5 \%$ of the genome encodes proteins (10-12). The functional complexity of organisms also seems to be associated with noncoding RNA (ncRNA) molecules. It is well established that ncRNAs, such as miRs (approx-

Authorship note: $Y Z, S X$, and $Y X$ contributed equally to this work. Conflict of interest: The authors have declared that no conflict of interest exists. Submitted: October 9, 2017; Accepted: July 26, 2018.

Reference information: J Clin Invest. 2018;128(10):4510-4524.

https://doi.org/10.1172/JCI97965 imately 21-25 nucleotides), play important roles in regulating synovial inflammation and proliferation in RA (13-15). However, miRs comprise only a small part of the noncoding regions of the mammalian genome, and other ncRNAs, such as long noncoding RNAs (lncRNAs), exhibit more abundant expression (16). lncRNAs, defined as long transcripts (>200 nucleotides) with no protein-coding potential, play important roles in regulating a series of cellular processes. For instance, lincRNA-p21 regulates the Warburg effect by repressing the p53 transcriptional pathway (17). IncRNA-HOX transcript antisense intergenic RNA (HOTAIR) is involved in adipocyte differentiation (18). lncRNAs play widespread roles in cell-cycle control (19). IncLSTR modulates systemic lipid metabolism via the TAR DNA-binding protein 43 (TDP-43)/ farnesoid $\mathrm{X}$ receptor (FXR)/apolipoprotein $\mathrm{C} 2$ pathway in mice (20). lnc-DC modulates DC differentiation by binding to STAT3 (21). IncRNAs also participate in epigenetic regulation (22-24) and embryonic vertebrate development (25). Moreover, increasing evidence suggests that abnormal expression or mutation of lncRNAs is involved in the pathogeneses of an array of human diseases, including cancer (26-29), diabetes mellitus (30), and cardiovascular disease (31). Although recent studies indicate that lncRNAs are involved in some autoimmune inflammatory diseases $(32,33)$, whether they also play a role in RA is largely unknown.

In the present study, we identified a lncRNA that has lowerthan-normal expression levels in rheumatoid fibroblast-like synoviocytes (LERFS; AK309896) and demonstrated its essential regulatory role in the aggressive and proliferative behavior of RA FLSs. LERFS functions through interactions with heterogeneous nuclear ribonucleoprotein Q (hnRNP Q), an RNA-binding protein that modulates mRNA metabolism (34-37) and controls cell motility by regulating mRNA translation of the small GTPase proteins CDC42 and RhoA $(38,39)$. We also observed that forma- 
tion of the LERFS-hnRNP Q complex is required for hnRNP Q to bind to RhoA, Rac1, and CDC42 mRNA, thereby negatively regulating their protein expression. Our findings suggest that LERFS is an important regulator of FLS proliferation and migration and that a decrease in LERFS expression might contribute to synovial aggression and proliferation in RA.

\section{Results}

IncRNA LERFS has lower-than-normal expression in FLSs and synovial tissues from patients with $R A$. To determine the expression pattern of lncRNA in RA, we used a microarray to establish lncRNA expression profiles in FLSs isolated from RA patients and healthy controls (HCs). Hierarchical clustering analyses showed distinguishable lncRNA expression patterns. Using an absolute fold change of at least 2.0 and a $P$ value of less than 0.05 , we observed that the expression of 94 lncRNAs was significantly upregulated and that the expression of $195 \operatorname{lncRNAs}$ was downregulated in the RA group compared with the HC group (Figure 1A, left, and Figure 1B). We found that 25 lncRNAs were downregulated in RA FLSs by more than 20-fold (Figure 1A, right).

In this study, we focused on the IncRNA LERFS, which is encoded by a gene at position chromosome 9q13 in the genome. A schematic graphic of the genomic locus of LERFS is shown in Supplemental Figure 1; supplemental material available online with this article; https:/doi.org/10.1172/JCI97965DS1). Quantitative reverse transcription PCR (RT-qPCR) confirmed the significant decrease in LERFS expression in RA FLSs compared with that in HC FLSs (Figure 1C). As proinflammatory mediators and growth factors play important roles in regulating RA FLS biological functions, we therefore observed the effects of TNF- $\alpha$, IL-1 $\beta$, IL-17, PDGF subunit B (PDGF-BB), and LPS on LERFS expression by highly expressed LERFS-RA FLSs. We found that only PDGFBB treatment induced significant downregulation of LERFS (Figure 1D). We also found that treatment with synovial fluid from patients with RA reduced LERFS expression, however, addition of a PDGF-neutralizing antibody (50 ng/ml, AB-220-NA; R\&D Systems) largely reversed LERFS expression (Figure 1D). Since a previous report showed that PDGF contributes to the proliferation and aggressive activity of RA FLSs (40), our data hint that LERFS might be associated with the proliferation and invasion of RA FLSs. We also observed that treatment of FLSs with methotrexate (MTX), an anchor drug for RA treatment, increased the expression of LERFS (Figure 1E), however, treatment with the potent antiinflammatory mediator dexamethasone (DXM) did not influence LERFS expression (Figure 1F). Using 5'- and 3'-RACE (rapid amplification of cloned cDNA ends), we determined LERFS to be a 1,729-nucleotide transcript with a poly (A) tail (Supplemental Figure 2). Analysis of its coding potential strongly suggested that LERFS is a ncRNA (Supplemental Figure 3). FISH using confocal microscopy showed that LERFS is located primarily in the cytoplasm (Figure 1G), which was confirmed by nuclear and cytoplasmic fractionation (Supplemental Figure 4); this indicates that LERFS might perform its biological function in the cytoplasm. Furthermore, to test for specificity of the LERFS probe used in FISH, we performed FISH in LERFS-silenced HC FLSs. We knocked down LERFS in HC FLSs by using a specific mixture of siRNA and an antisense oligonucleotide (ASO) (Supplemental Figure
5). We found that LERFS expression was significantly reduced in LERFS-silenced HC FLSs. As a positive control, we also detected LERFS expression levels in LERFS-overexpressed RA FLSs (Figure $1 G)$. Moreover, RNA ISH of paraffin-embedded synovial tissue (ST) sections using ISH staining confirmed the expression of LERFS, which was decreased in patients with established RA compared with expression levels in HCs, however, LERFS expression was increased in synovium from RA patients in remission (Figure $1 \mathrm{H})$. These data indicate that decreased expression of synovial LERFS may be involved in RA pathogenesis.

IncRNA LERFS is a negative regulator of RA FLS migration and invasion. To explore the potential role of LERFS in RA, we increased LERFS expression using lentivirus-based overexpression of lncRNA in primary RA FLSs (Supplemental Figure 6). To determine the regulation of LERFS in directed migration, we detected the chemotaxis migration of FLSs using a Transwell chamber assay in lentivirus-mediated, lncRNA-overexpressed RA FLSs. We found that RA FLSs with LERFS overexpression had decreased FBSinduced migration compared with the empty vector control (Figure 2A). We also observed the inhibitory effect of LERFS overexpression on the migration of RA FLSs induced by $50 \mathrm{ng} / \mathrm{ml}$ monocyte chemoattractant protein 1 (MCP-1), one of the important chemokines in RA (Supplemental Figure 7). We further used a monolayer wound-scratch assay to determine the role of LERFS in cell migration. As shown in Figure 2C, RA FLSs with overexpression of LERFS displayed significantly lower cell migration compared with empty vector control. However, we determined that the migration of HC FLSs with LERFS overexpression was not different from that of the empty vector control (Figure 2, B and D).

Aggressive destruction of cartilage and bone is a key pathogenic behavior of RA FLSs; the in vitro invasion potential of RA FLSs is well associated with the rate of joint destruction in patients with RA (41). Therefore, we determined the effect of LERFS overexpression on modulating invasion by RA FLSs of Matrigel-coated Transwell membranes. We observed that LERFS overexpression reduced such invasion compared with results for the empty vector control (Figure 2E). Consistent with our finding for migration, LERFS overexpression also did not affect the invasion of HC FLSs (Figure $2 \mathrm{~F}$ ). Collectively, our findings indicate that the role of LERFS might be more pronounced in RA FLSs than in HC FLSs. Additionally, we determined the effect of LERFS overexpression on the expression of MMP-1, MMP-3, and MMP-13, however, LERFS overexpression did not affect their expression (data not shown).

In order to rule out the impact of proliferation, we assessed the growth rate of FLSs in serum-free medium at 0,24 , and 48 hours and found that the cell growth rate did not differ among these time points (Supplemental Figure 8). Furthermore, we found that addition of a proliferation inhibitor, mitomycin, did not affect the migration or invasion of these cells (Supplemental Figure 9). Therefore, these data eliminate the possibility that changes seen in migration and invasion are due to changes in the proliferation of RA FLSs.

Dynamic reorganization of the actin cytoskeleton is essential for optimal cell migration. To determine the role of LERFS in modulating actin reorganization in RA FLSs, we used fluorescent phalloidin staining to visualize polymerized actin in PDGF-BBinduced migrating cells after wounding in overexpressed LERFSor empty vector control-transfected RA FLSs. As shown in Figure 
A
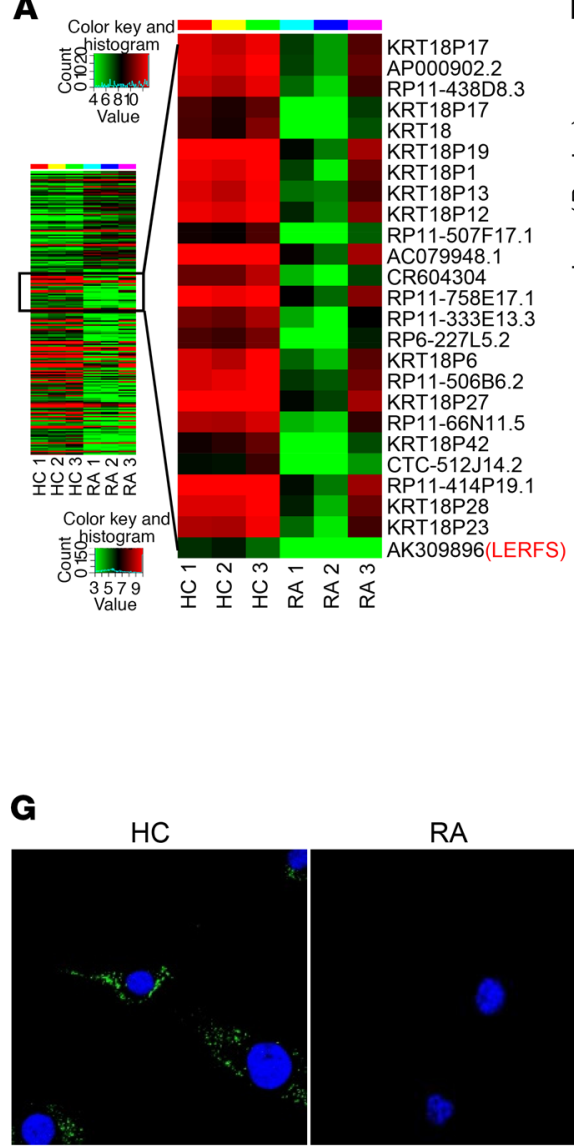

LERFS

$\mathrm{HC}+\mathrm{siC}$

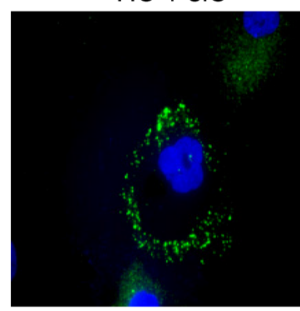

LERFS

H

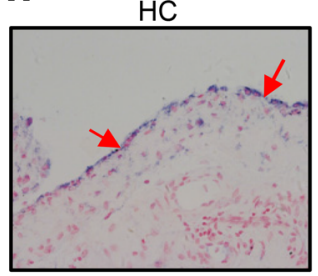

LERFS

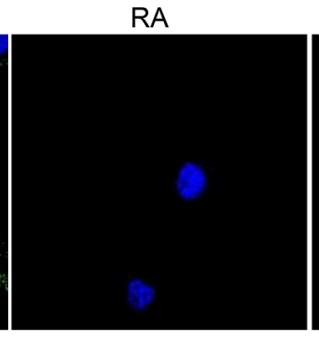

LERFS

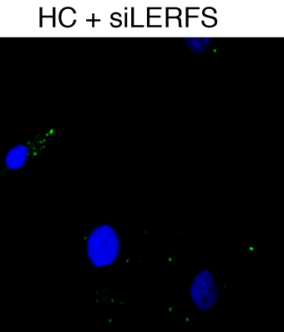

LERFS

RA

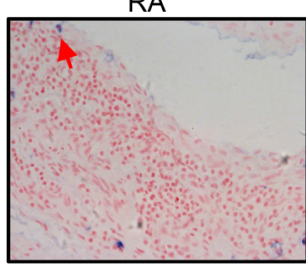

LERFS
B

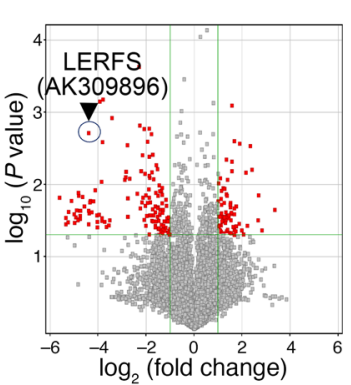

E

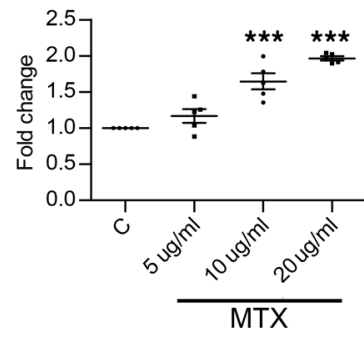

D
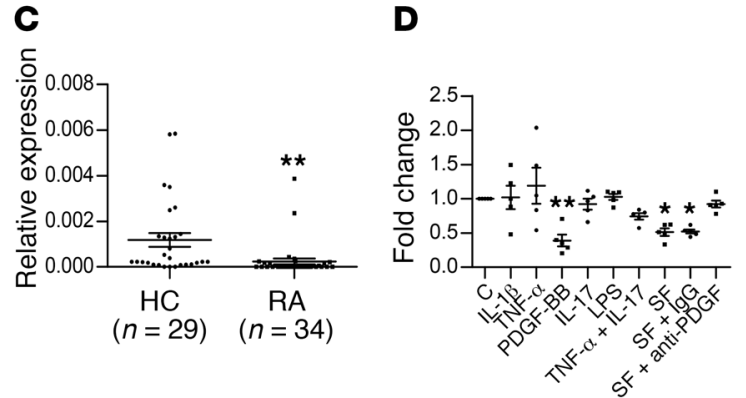

$\mathbf{F}$

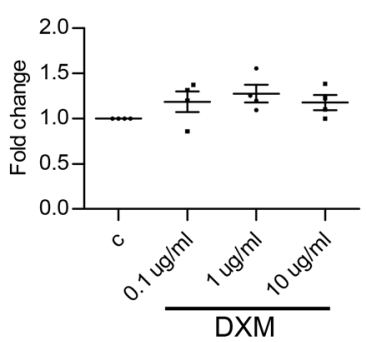

RA
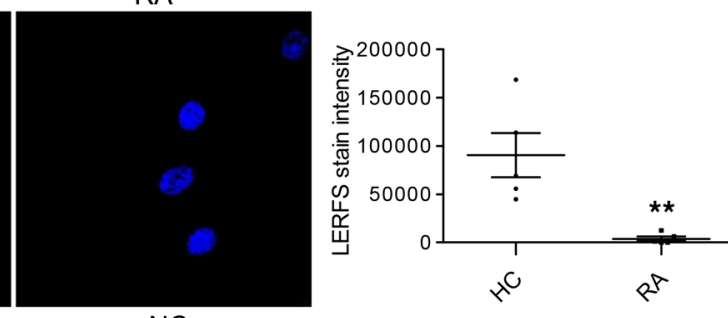

NC

$\mathrm{RA}+$ Vector

RA + LERFS OE

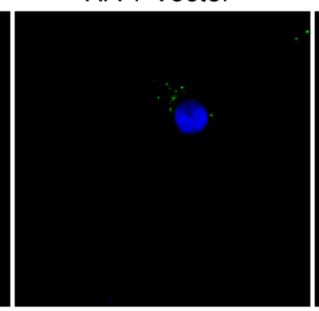

LERFS

$\mathrm{RA}$ in remission

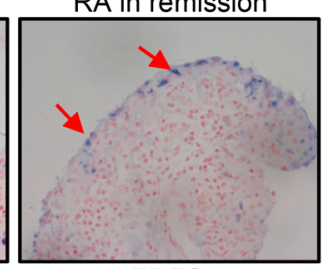

LERFS
LERFS

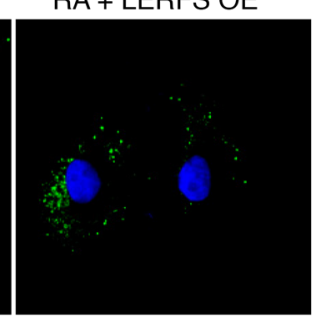

RA

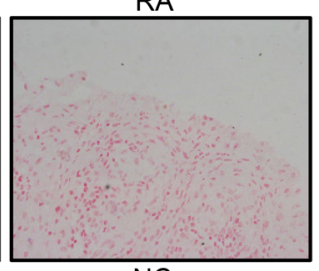

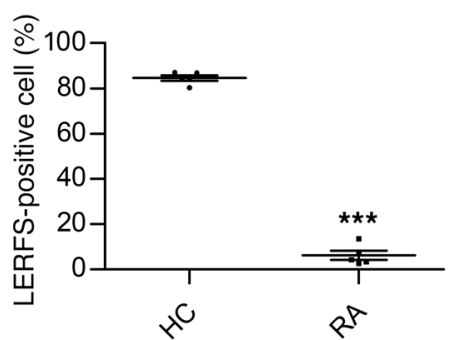


Figure 1. Decreased levels of LERFS IncRNA in FLSs and STs from

patients with RA. (A) Total RNA harvested from RA FLSs $(n=3)$ and HC FLSs $(n=3)$ was screened by microarray analysis. Microarray heatmap of differentially expressed IncRNAs. (B) Volcano plot shows differentially expressed IncRNAs between RA FLSs and HC FLSs. $P<0.05$, by Student's $t$ test. (C) The expression level of LERFS was validated by RT-qPCR in HC FLSs $(n=29)$ and RA FLSs $(n=34)$. Ct values were normalized to GAPDH. Data are presented as the mean $\pm \mathrm{SEM}$. ${ }^{* *} P<0.01$ versus $\mathrm{HCs}$, by Student's $t$ test. (D) RA FLSs were stimulated with IL-1 $1 \beta(10 \mathrm{ng} / \mathrm{ml})$, TNF- $\alpha$ (10 $\mathrm{ng} / \mathrm{ml})$, PDCF-BB (10 ng/ml), IL-17 (10 ng/ml), LPS (10 ng/ml), synovial fluid (SF), or synovial fluid containing IgC (SF + IgG) or a PDGF-neutralizing antibody (SF + anti-PDCF) $(50 \mathrm{ng} / \mathrm{ml})$ for 24 hours. (E and F) RA FLSs were treated with MTX (E) or DXM (F) for 24 hours. (D-F) ${ }^{*} P<0.05,{ }^{* *} P$ $<0.01$, and ${ }^{* * *} P<0.001$ versus untreated control, by 1-way ANOVA with Bonferroni's post hoc comparison $(n=5)$. (G) Localization of LERFS was evaluated by RNA FISH assay. For silencing of LERFS, HC FLSs were transfected with a specific mixture of siRNA and ASO for LERFS (siLERFS). Shown are representative images of LERFS (green) and nuclei (blue). Graph shows the quantification of staining intensity for 5 different RA patients and HCs. Original magnification, $\times 630 .{ }^{*} P<0.01$ versus $H C s$, by Student's $t$ test. (H) LERFS expression, detected by ISH staining, on STs from HCs and RA patients. Shown are representative images and quantification of the percentage of LERFS-positive cells for 5 different RA patients or HCs. Also shown is a representative image of $R A$ in remission from 2 remitted patients treated with MTX and TNF- $\alpha$ inhibitor. A scrambled probe was used as a NC. Red arrows indicate LERFS-positive (blue) cells. Original magnification, $\times 400 .{ }^{* *} P<0.001$ versus $\mathrm{HCs}$, by Student's $t$ test. C, control. Data are presented as the mean \pm SEM.

$2 G$, FLSs transfected with the empty vector control displayed flat or ruffling lamellipodia and filopodia at their leading edges, while cells with overexpressed LERFS had reduced lamellipodia and filopodia formations. This suggests that LERFS is involved in the formation of membrane protrusions in migrating cells.

To further confirm an important role of LERFS in cell mobility, we observed the effect of LERFS knockdown on FLS migration and invasion. We demonstrated that, when compared with negative controls (NCs), HC FLSs with LERFS knockdown showed increased chemotaxis migration (Figure $2 \mathrm{H}$ ) and invasion of Matrigel-coated Transwell membranes (Figure 2I).

Finally, we determined the effect of LERFS overexpression on in vivo migration of and invasion by RA FLSs. We intradermally implanted RA FLSs into nude mice and then measured migrated FLSs using IHC staining with anti-human class I HLA antibody. As shown in Figure 2J, LERFS overexpression reduced the ability of RA FLSs to migrate in vivo. To evaluate the in vivo effect of LERFS overexpression on the invasion of RA FLSs into cartilage, we used the SCID mouse coimplantation model. We coimplanted RA FLSs carrying overexpressed LERFS or empty vector side by side into the left or right flanks of SCID mice. As shown in Figure 2K, RA FLSs transfected with overexpressed LERFS exhibited a significant reduction of invasion into cartilage as compared with the cells transfected with empty vector. Taken together, our data suggest that LERFS negatively modulate aggression in RA FLSs.

LERFS represses the proliferation of RA FLSs. Abnormal proliferation and apoptosis of resident FLSs are considered to be important contributors to rheumatoid synovial hyperplasia, eventually initiating the destructive phase of the disease. Therefore, we explored the role of LERFS in regulating the proliferation and apoptosis of RA FLSs. We first observed that lentiviral
LERFS overexpression reduced RA FLS proliferation compared with the empty vector control (Figure 3A). The results from the MTT assay also showed that LERFS overexpression decreased cell proliferation (Figure 3B). However, we found that the proliferation of HC FLSs with LERFS overexpression was not different with that of the empty vector control (Figure 3C). Moreover, analysis of the different phases of the cell cycle after transfection with lentivirus-overexpressed LERFS showed cell-cycle arrest at the $G_{2} / M$ phase (Figure 3, D and E). To rule out influence of the heterogeneity of RA FLSs from different patients, we used the RA FLS cell line MH7A to evaluate the effect of LERFS overexpression on the cell cycle. We also assessed the effect of LERFS overexpression on cell-cycle arrest at the $G_{2} / M$ phase in the MH7A cell line (Supplemental Figure 10).

In addition, we observed the effect of LERFS knockdown on the proliferation of FLSs. When compared with NCs, we found that LERFS knockdown in HC FLSs resulted in increased proliferation (Figure 3F). These data further support the notion of an important role for LERFS in regulating cell proliferation.

Next, we evaluated the effect of LERFS overexpression on apoptosis of RA FLSs, using APC annexin V and 7-AAD staining to detect apoptosis of RA FLSs by flow cytometry. We observed that the total apoptotic cell population did not increase significantly in cells with overexpressed LERFS compared with the empty vector control cells (Figure 3G), nor did we find any effect of LERFS overexpression on caspase 3 or caspase 7 activity (Figure 3H). We also determined that LERFS overexpression did not influence death receptor-mediated (FasL-mediated) apoptosis (Figure 3I). Collectively, these data suggest that decreased LERFS expression contributes to the proliferation, but not apoptosis, of RA FLSs.

IncRNA LERFS interacts with hnRNP $Q$ to regulate the migration, invasion, and proliferation of RA FLSs. IncRNAs usually function by physically binding to other cellular factors (11-13, 17, 18). To evaluate how LERFS regulates the migration, invasion, and proliferation of RA FLSs, we sought to identify intracellular LERFS-binding factors using an RNA-pulldown assay. We transcribed full-length LERFS in vitro with biotinylated nucleotides, incubated the biotinylated LERFS (or antisense LERFS as a NC) with total cell lysates from high LERFS-expressing RA FLSs, and pulled them down with streptavidin. The related proteins were resolved by SDS-PAGE and visualized by silver staining (Figure 4A). We excised 1 distinct band specifically present in the LERFS-pulldown samples (Figure 4B) and analyzed it using mass spectrometry (MS). Interestingly, hnRNP $\mathrm{Q}$ was identified as the only pulled-down protein with the potential to bind to LERFS. To confirm that hnRNP $Q$ binds specifically to LERFS, we repeated the pulldown assay with biotinylated LERFS and probed for hnRNP Q using immunoblot analysis. We obtained a similar result, in that hnRNP Q bound specifically to LERFS (Figure 4C).

To corroborate these findings, we used an anti-hnRNP Q antibody to immunoprecipitate endogenous hnRNP $\mathrm{Q}$ from total lysates of RA FLSs expressing high levels of LERFS and then extracted and analyzed the RNA bound to hnRNP Q. We observed a greater than 3-fold enrichment of LERFS in the anti-hnRNP Q immunoprecipitates compared with that detected in the IgG control (Figure 4D). We also observed binding of LERFS to hnRNP 
A

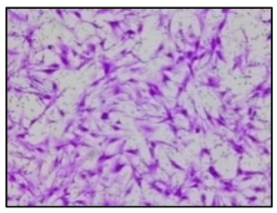

C

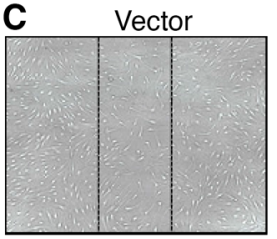

E

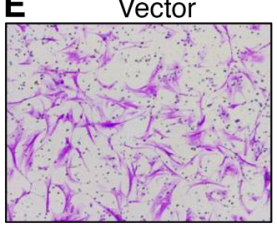

G

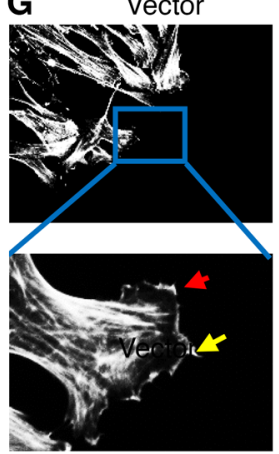

J

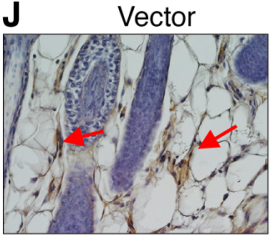

LERFS OE

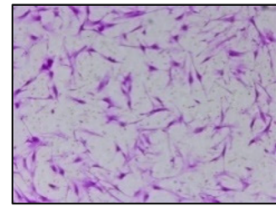

LERFS OE

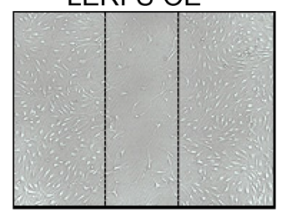

LERFS OE

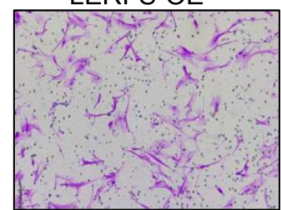

LERFS OE

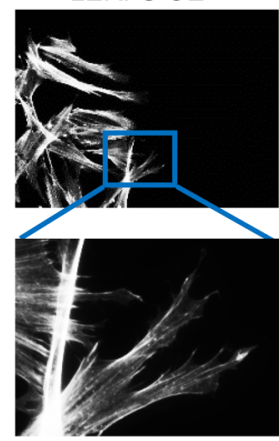

LERFS OE

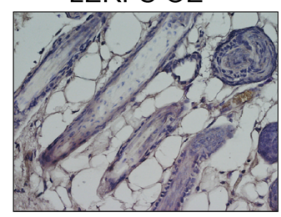

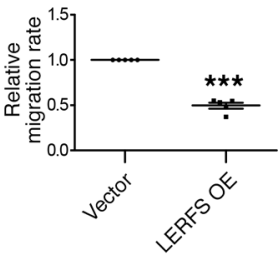

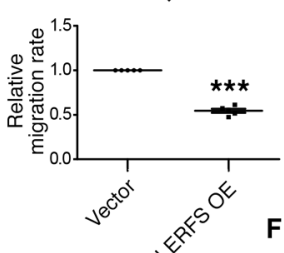

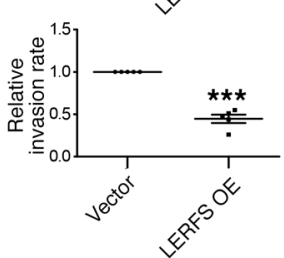

$\mathbf{F}$

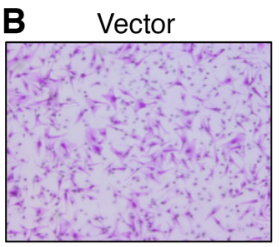

D Vector

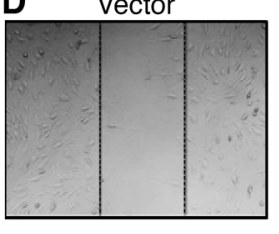

F Vector

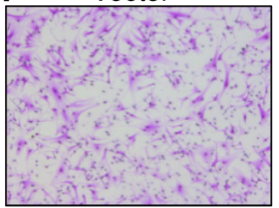

H $\quad \mathrm{siC}$
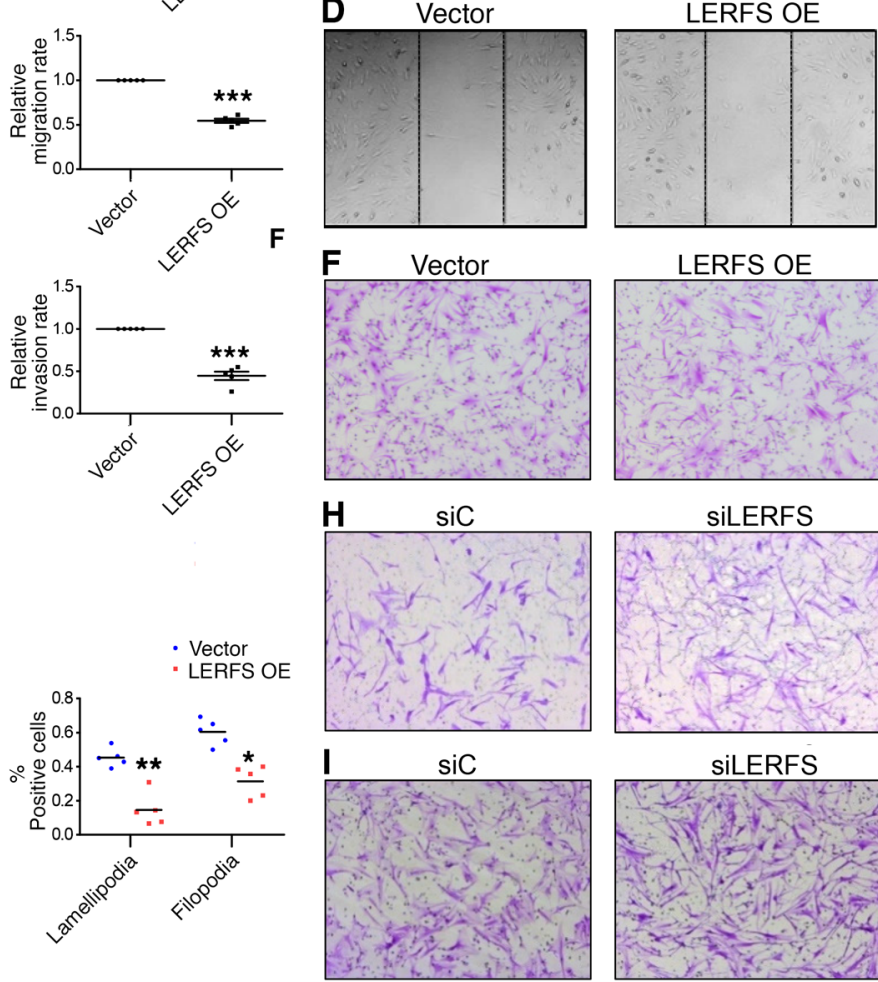

LERFS OE

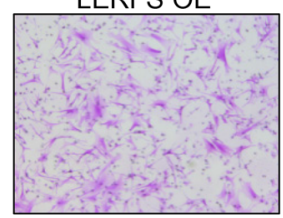

SiLERFS

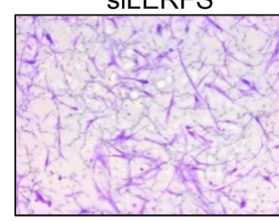

siLERFS
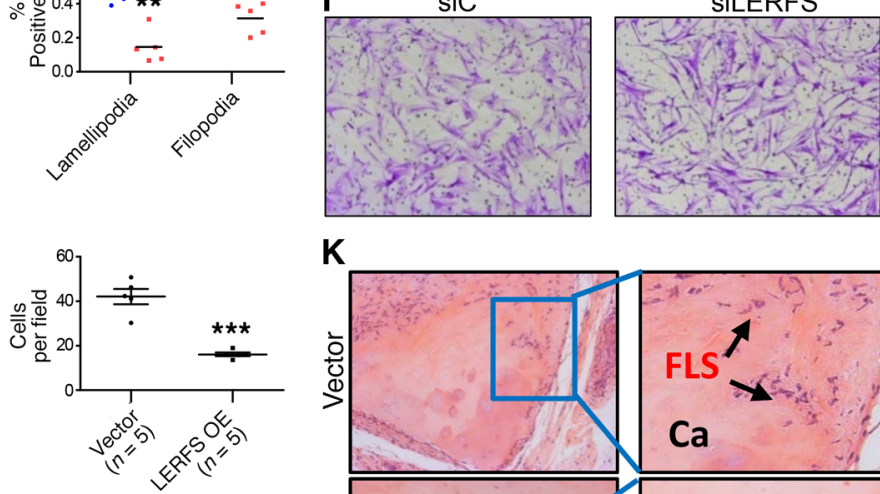

K

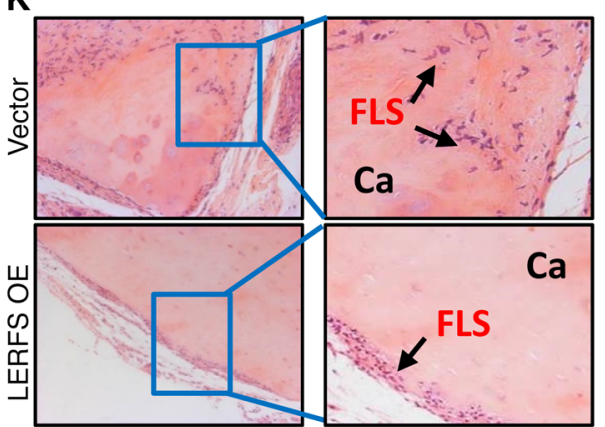

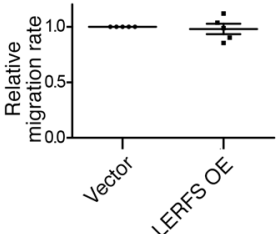
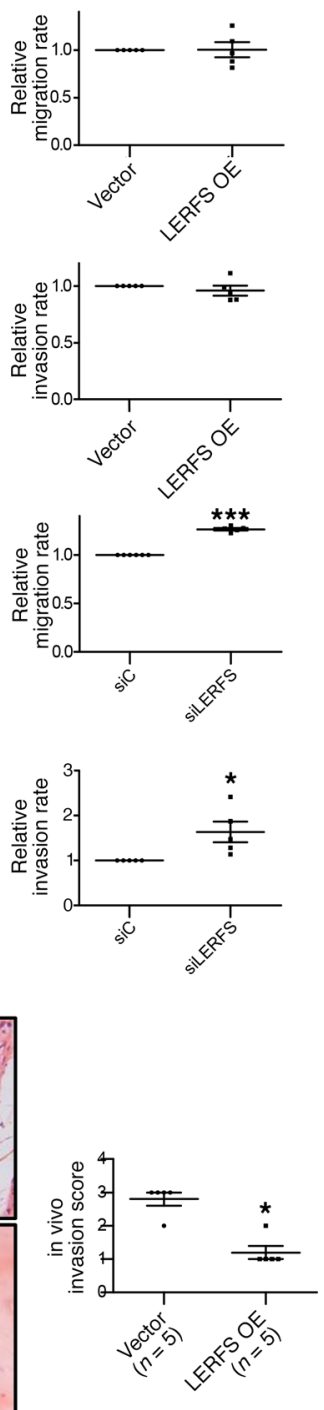

Figure 2. Inhibitory effects of LERFS overexpression on RA FLS migration and invasion. (A and B) Chemotaxic migration of RA FLSs (A) or HC FLSs (B) was evaluated using a Transwell assay. Representative images (original magnification, $\times 100$ ) are shown. Graphs indicate the relative migration rates. (C and D) The migration of RA FLSs (C) or HC FLSs (D) was analyzed using a wound-healing assay. Representative images are shown (original magnification, $\times 50$ ). The relative migration rate represents the number of migrated cells normalized to the vector control. (E and $\mathbf{F})$ In vitro invasion was determined using inserts coated with Matrigel Basement Membrane Matrix. The relative invasion rate was calculated by counting invaded cells and then normalized to the vector control. Representative images (original magnification, $\times 100$ ) are shown. Graphs indicate the relative invasion rates. (G) LERFS overexpression impaired the formation of pseudopodium in RA FLSs. RA FLSs were wounded and stimulated with PDGF-BB $(10 \mathrm{ng} / \mathrm{ml})$ for 4 hours. Representative images are shown. Original magnification, $\times 400$ (top); $\times 1,000$ (bottom). Red arrow indicates lamellipodia formation; yellow arrow indicates filopodia formation. Graph indicates the number of RA FLSs with positive lamellipodia or filopodia. (H and I) Images show that LERFS knockdown promoted HC FLS migration (H) and invasion (I). Original magnification, $\times 100$. Graphs indicate the relative migration $(\mathbf{H})$ and invasion $(\mathbf{I})$ rates. (J) Effect of LERFS overexpression on in vivo migration of RA FLSs. Representative images are shown (original magnification, $\times 400$ ); red arrows indicate human FLSs. Graph indicates the number of migrated human FLSs stained with anti-human class I HLA antibody. (K) Effect of LERFS overexpression on the invasion of RA FLSs into human cartilage implants transferred under the skin of SCID mice. Arrows indicate RA FLS invasion into cartilage (Ca). Original magnification, $\times 200$ (left); $\times 400$ right (enlarged). Graph indicates the invasion scores. Data are shown as the mean \pm SEM of 5 independent experiments involving 5 different RA patients or HCs. ${ }^{*} P<0.05$, ${ }^{*} P<0.01$, and ${ }^{* *} P<0.001$ versus vector control, by Student's $t$ test. 
A

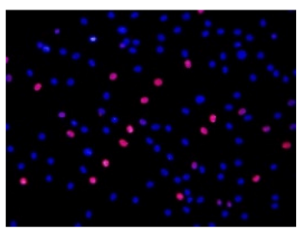

C Vector

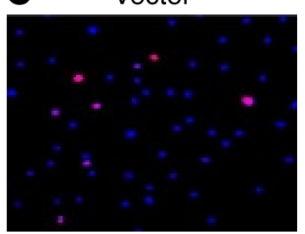

D

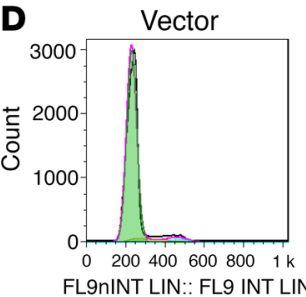

$\mathbf{F}$

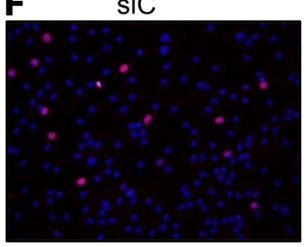

H

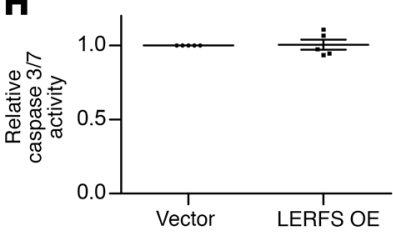

LERFS OE

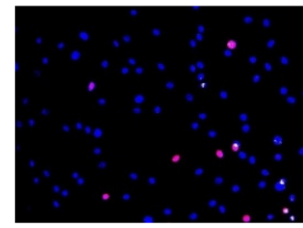

LERFS OE
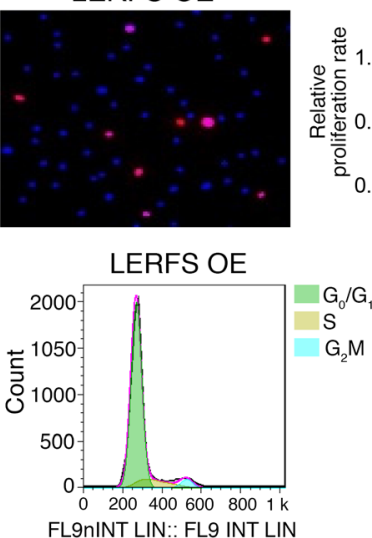

siLERFS

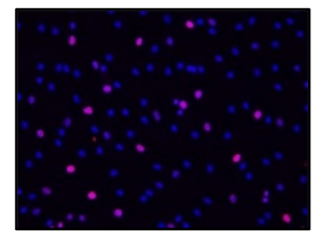

I

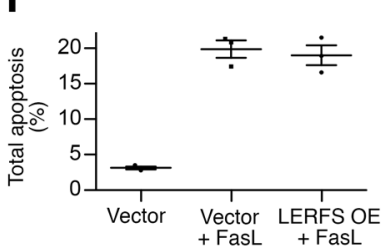

E
B
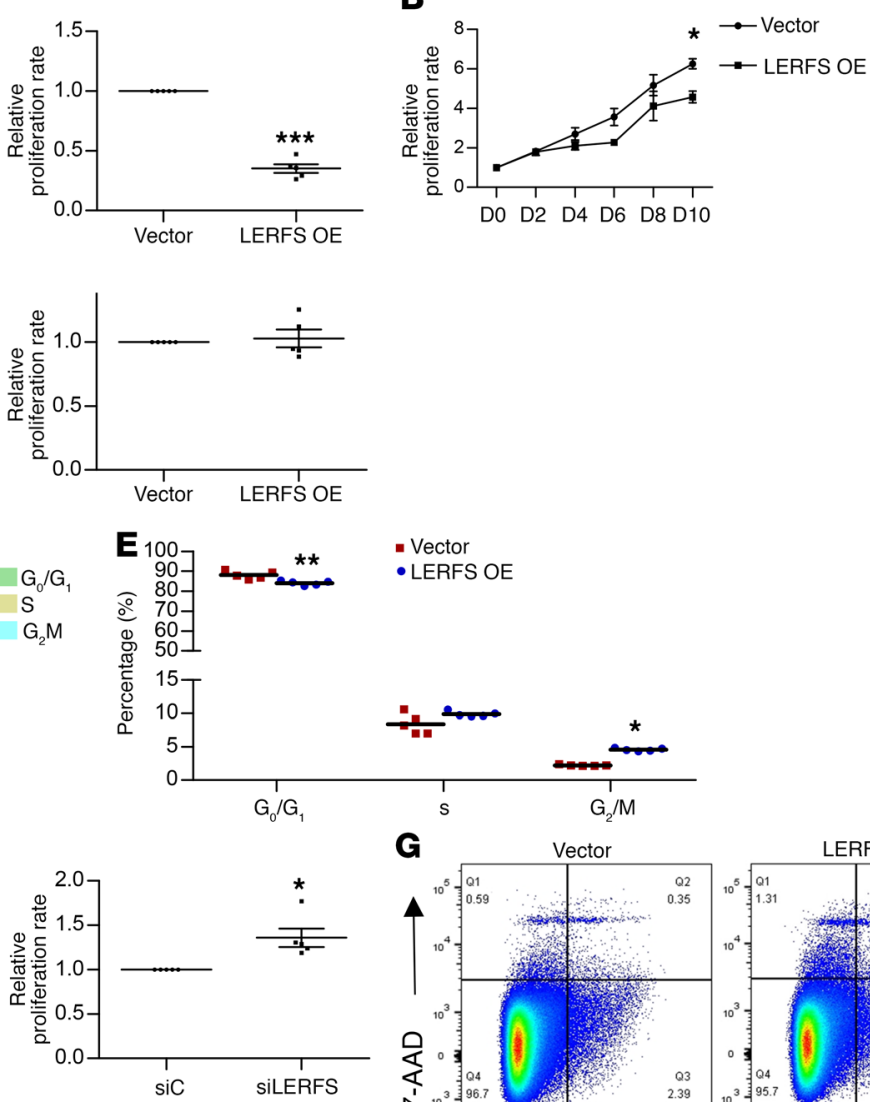

G
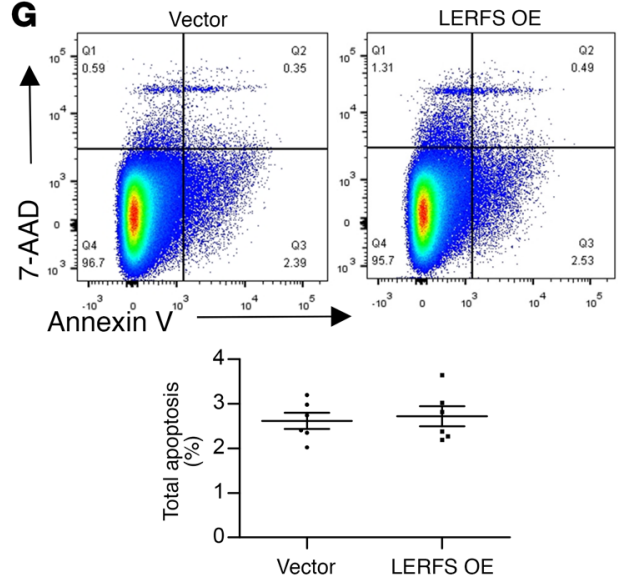

Figure 3. Effect of LERFS overexpression on the proliferation and apoptosis of RA FLSs. (A and B) An EdU incorporation assay was performed to evaluate cell proliferation. Representative images show proliferation of RA FLSs (A) and HC FLSs (C) labeled with EdU (red) and nuclei stained with Hoechst 33342 (blue) (original magnification, $\times 200$ ). Graphs in $\mathbf{A}$ and $\mathbf{C}$ indicate the mean \pm SEM of 5 independent experiments involving 5 different RA patients or HCs. (B) Detection of cell growth rates in vitro using an MTT assay at the indicated time points after lentivirus infection (DO indicates the day of infection). Values are expressed relative to DO as the mean \pm SEM of 5 independent experiments. ( $\mathbf{D}$ and $\mathbf{E}$ ) Effects of LERFS overexpression on phases of the cell cycle. (D) Representative plots of cell-cycle distribution. (F) LERFS knockdown promotes proliferation by HC FLSs. Representative images are shown (original magnification, $\times 200$ ). Data are shown as the mean \pm SEM of 5 independent experiments involving 5 different RA patients (E) or HCs (F). (C) Effect of LERFS overexpression on apoptosis of RA FLSs. The cellular apoptosis rate was measured by annexin $V$ and 7-AAD staining and detected by flow cytometry. Representative flow plots are shown. Total apoptosis represents the mean \pm SEM percentage of 5 independent experiments involving 5 different RA patients. (H) Quantitative measurement of caspase 3/7 activity. Data are expressed relative to vector values and presented as the mean \pm SEM of 5 independent experiments involving 5 different RA patients. (I) Effect of LERFS overexpression on FasL-induced apoptosis of RA FLSs. Cells were stimulated with or without $100 \mathrm{ng} / \mathrm{ml}$ recombinant human FasL for 24 hours. Total apoptosis represents the mean \pm SEM of 3 independent experiments involving 3 different RA patients. ${ }^{*} P<0.05,{ }^{* *} P<0.01$, and ${ }^{* * *} P<0.001$ versus vector, by Student's $t$ test.

Q in HC FLSs, which was evidenced by increased binding of the LERFS-hnRNP Q complex compared with that observed in RA FLSs (Figure 4E). Our observations confirmed that LERFS and hnRNP Q might form an RNP complex.

Next, we evaluated whether hnRNP Q helps modulate the migration, invasion, and proliferation of RA FLSs. We knocked down hnRNP Q expression using siRNA; to rule out nonspecific interference, we constructed 3 different sequences of siRNA oligonucleotides for hnRNP Q. As shown in Supplemental Figure 11, transfection with all 3 siRNA oligonucleotides knocked hnRNP Q mRNA levels down, but the inhibitory effect of hnRNP Q siRNA-3 was the strongest. Accordingly, we used hnRNP Q siRNA-3 (sihnRNP Q) for subsequent experiments. Interestingly, hnRNP Q knockdown resulted in significant increases in migration, invasion, and proliferation (Figure 4, F-H). Conversely, overexpression of hnRNP Q decreased migration, invasion, and proliferation (Figure 4, I-K). Our results suggest that hnRNP Q negatively regulates the migration, invasion, and proliferation of RA FLSs. 
A

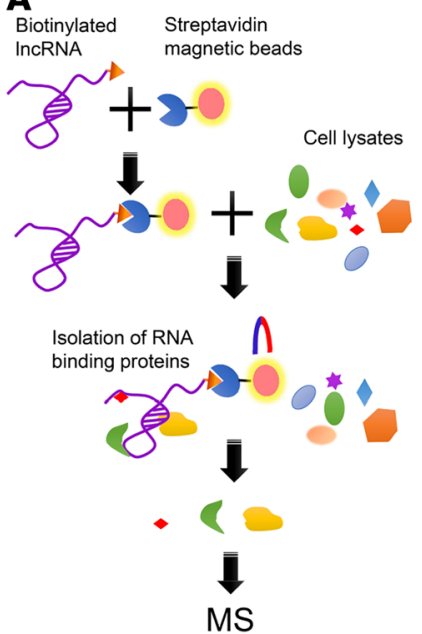

$\mathbf{F}$

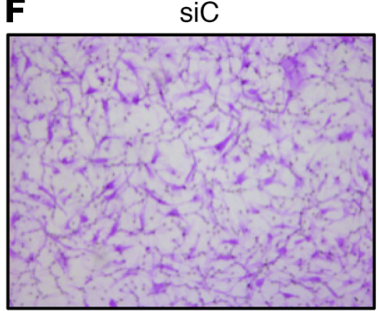

G
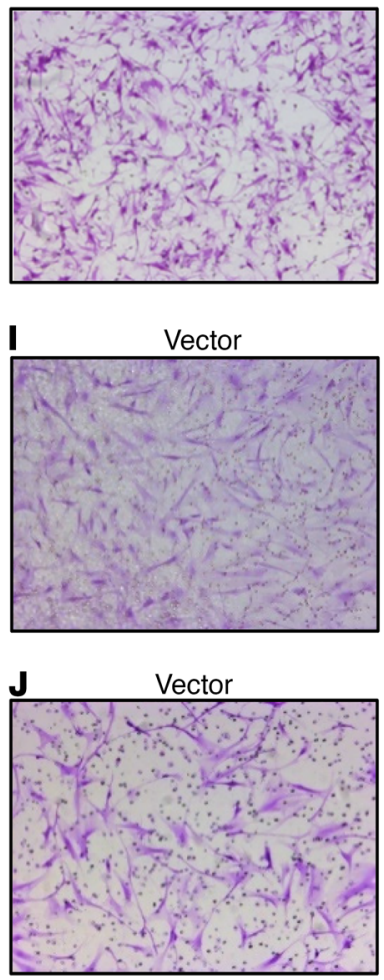

B
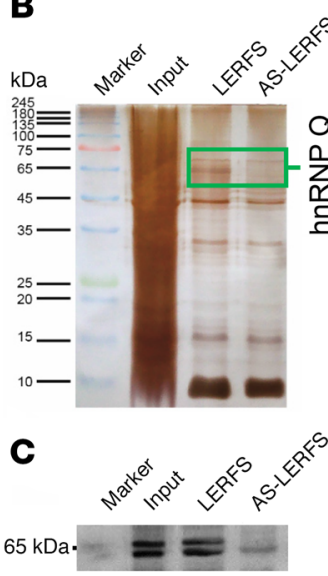

sihnRNP Q
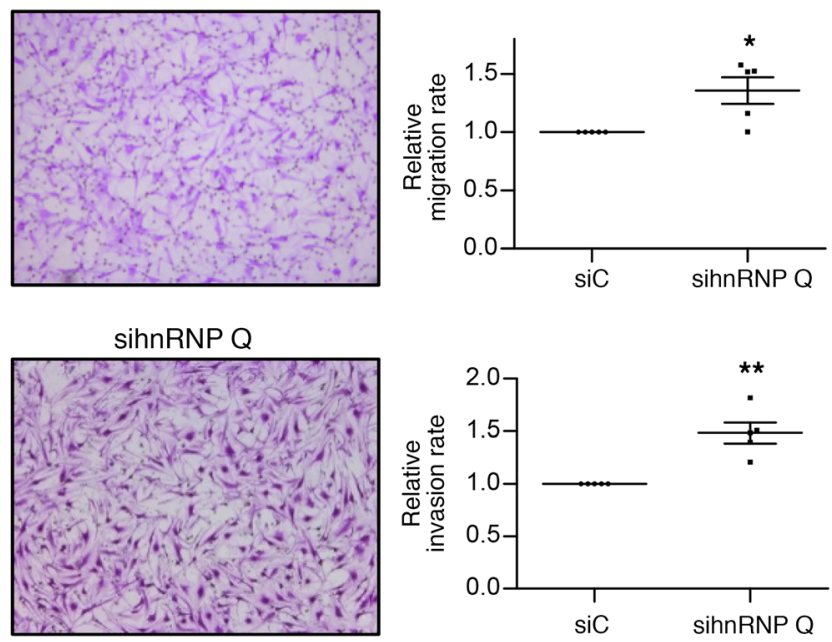

HnRNP Q1 OE
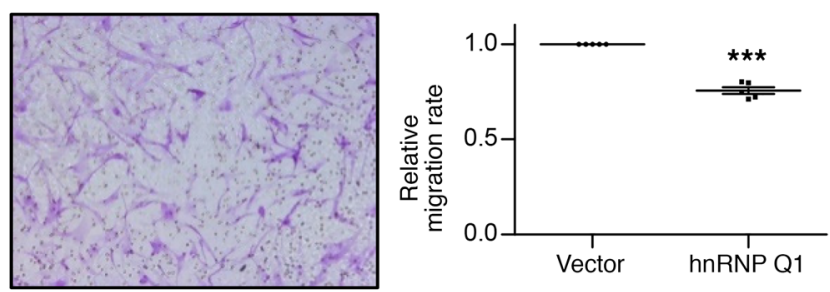

HnRNP Q1 OE

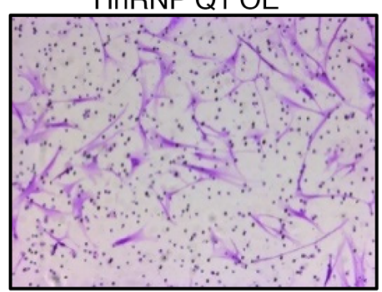

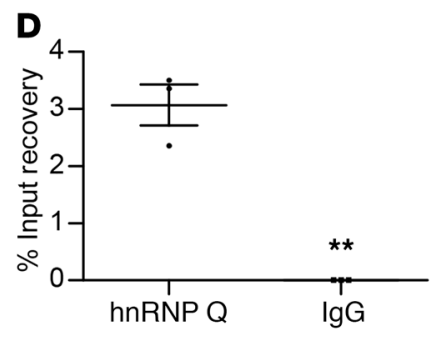
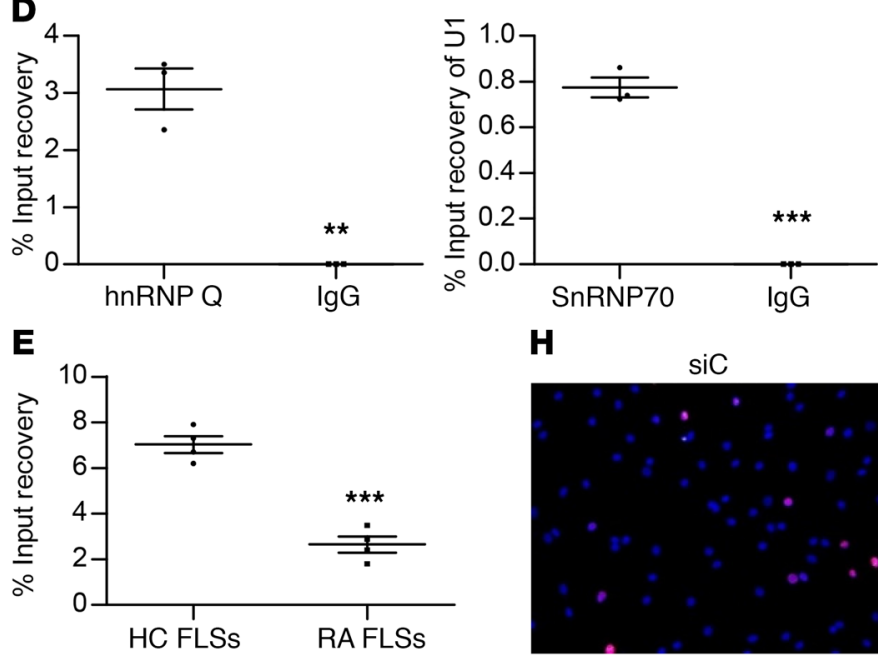

H

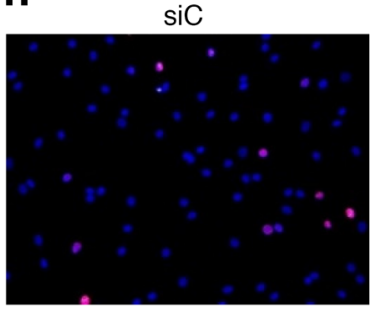

sihnRNP Q
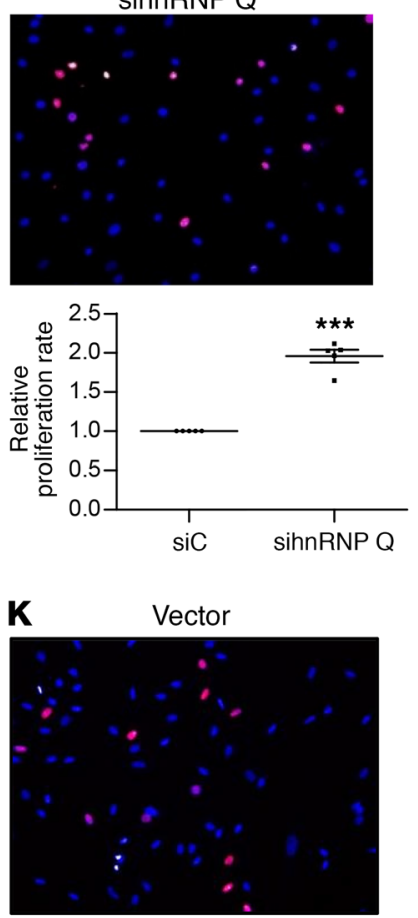

hnRNP Q1 OE
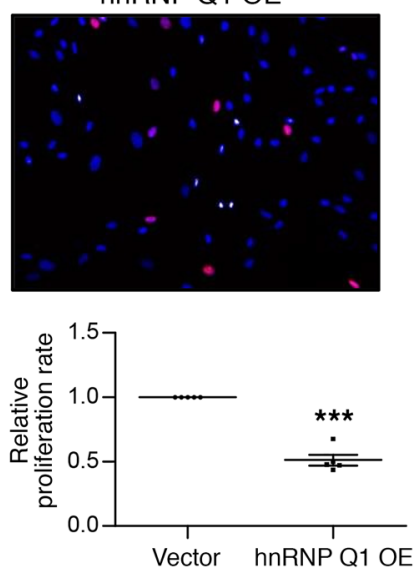
Figure 4. LERFS functions by interacting with hnRNP Q. (A) Experimental design for pulldown assays and identification of LERFS-associated cellular proteins. LERFS RNA was biotinylated by in vitro transcription, refolded, and incubated with lysates of RA FLSs. (B) Silver staining of biotinylated LERFS-associated proteins. A LERFS-specific band was excised and analyzed by MS, which identified hnRNP Q. (C) Western blot of proteins from LERFS-pulldown assays. (D) RIP evaluation of the interaction between hnRNP $Q$ and LERFS within RA FLSs using an anti-hnRNP $Q$ antibody $(5 \mu \mathrm{g})$, with IgG ( $5 \mu \mathrm{g})$ as a NC. SnRNP70 was used as a positive control (right). ${ }^{* *} P<0.01$ and ${ }^{* *} P<0.001$ versus IgG, by Student's $t$ test.U1, U1 small nuclear RNA (snRNA). (E) Comparison of LERFS binding with hnRNP $Q$ between RA FLSs and HC FLSs. Data are shown as the mean \pm SEM of 3 independent experiments involving 3 different RA patients and $\mathrm{HCs}$. ${ }^{* *} P<0.001$ versus HC FLSs, by Student's $t$ test. $(\mathbf{F}-\mathbf{H})$ Effect of hnRNP $Q$ knockdown on the migration, invasion, and proliferation of RA FLSs. Representative images are shown (original magnification, $\times 200$ ). Data for relative migration $(\mathbf{F})$, invasion $(\mathbf{C})$, and proliferation $(\mathbf{H})$ are shown as the mean \pm SEM of 5 independent experiments involving 5 different RA patients. ${ }^{*} P<0.05,{ }^{* *} P<0.01$, and ${ }^{* *} P<0.001$ versus siControl (siC) or vector, by Student's $t$ test. (I-K) Overexpression of hnRNP Q suppressed the migration (I), invasion (J), and proliferation (K) of RA FLSs. Representative images are shown. Original magnification, $\times 100$ (I and J); $\times 200$ (K). Data in I-K were normalized to the control group (vector) and are presented as the mean \pm SEM of 5 independent experiments involving 5 different RA patients. ${ }^{* *} P<0.01$ and ${ }^{* *} P<0.001$ versus siC or vector, by Student's $t$ test.

LERFS and hnRNP Q coregulate Rho GTPase protein expression in RA FLSs. hnRNP Q is an RNA-binding protein with functions in mRNA metabolism that are localized in both the cytoplasm and nuclei. Our data show that LERFS is localized in the cytoplasm and suggest that LERFS might bind to cytoplasmic hnRNP Q in FLSs. Previous studies have indicated that hnRNP Q is involved in regulating mRNA metabolism of the small GTPases RhoA and CDC42 $(38,39)$ and that Rho family proteins play important roles in modulating RA FLS migration, invasion, and proliferation (41-43). Thus, we speculate that Rho family proteins might mediate the actions of LERFS and hnRNP Q in modulating the migration, invasion, and proliferation of RA FLSs. We observed that overexpression of LERFS downregulated Rac1 mRNA expression but did not affect the mRNA expression of RhoA or CDC42 (Figure 5A). Interestingly, we showed that LERFS overexpression reduced the protein expression and activation of RhoA, Rac1, and CDC42 (Figure 5, $B$ and $C$ ). Consistent with what we observed with LERFS, hnRNP Q knockdown by siRNA increased Rac1 mRNA expression, but it did not affect mRNA expression of RhoA or CDC42 (Figure 5D), and it increased the protein expression and activation of RhoA, Rac1, and CDC42 (Figure 5E and F). Moreover, hnRNP Q overexpression reduced the mRNA expression of Rac1 but not of RhoA or CDC42 (Supplemental Figure 12). These data suggest that LERFS and hnRNP Q regulate Rho protein expression through different modes of action. In other words, LERFS and hnRNP Q might modulate the stability or translation of Rac1 mRNA but might regulate only the mRNA translation of RhoA and CDC42.

Next, we sought to discover the mode of action of LERFShnRNP Q-Rho proteins. We first wondered whether LERFS could modulate the expression of hnRNP Q. Western blot analysis showed that LERFS overexpression did not affect hnRNP Q expression (Figure 5G). The RNA immunoprecipitation (RIP) assay showed that hnRNP Q could bind RhoA, Rac1, and CDC42 mRNAs
(Figure 5H), which was promoted by the overexpression of LERFS (Figure 5I). Furthermore, hnRNP Q knockdown by siRNA reversed the LERFS overexpression-induced reduction of RhoA, Rac1, and CDC42 protein expression and activity (Figure 5, J and K). We also observed that the translation inhibitor cycloheximide inhibited the hnRNP Q-knockdown-induced increase in protein expression of RhoA, Rac1, and CDC42 in LERFS-overexpressed RA FLSs (Supplemental Figure 13). Furthermore, we found that the LERFS overexpression-induced decrease in RA FLS migration, invasion, and proliferation was reversed by knockdown of hnRNP Q (Supplemental Figure 14). Intriguingly, bioinformatics analysis revealed no Alu element in the LERFS sequence, suggesting that LERFS could not bind directly to the mRNAs of RhoA, Rac1, or CDC42. Therefore, our results indicate that LERFS regulates the protein expression of RhoA, Rac1, and CDC42 through its interaction with hnRNP Q in the cytoplasm, forming a cytoplasmic LERFS-hnRNP Q complex, which then anchors to RhoA, Rac1, and CDC42 to decrease the stability or translation of target mRNAs.

\section{Discussion}

In this study, we identified lncRNA LERFS in FLSs and defined its decreased expression in RA FLSs and its function as a negative regulator in the migration, invasion, and proliferation of RA FLSs. In HC FLSs, LERFS bound with cytoplasmic hnRNP Q to form an RNA-protein complex, which further anchored to the target mRNAs of RhoA, Rac1, and CDC42. This interaction reduced the stability or translation of mRNAs and downregulated their protein levels. Conversely, in RA FLSs, decreased expression of LERFS resulted in reduced formation of the LERFS-hnRNP Q complex, which prevented binding of hnRNP Q to target mRNAs and thereby increased the stability or translation of mRNAs and upregulated their protein levels (Figure 6).

A recent study detected an abnormal expression pattern of some lncRNAs in RA $(44,45)$, but their contribution to the pathogenesis of RA is still unknown. In our study, we identified the lncRNA LERFS, which we found to have lower-than-normal expression levels in FLSs and STs from patients with RA. To evaluate the role of LERFS in RA, we performed overexpression and knockdown experiments. Overexpression of LERFS reduced the migration, invasion, and proliferation of RA FLSs, and knockdown of LERFS increased the migration, invasion, and proliferation of HC FLSs. These data imply that LERFS negatively regulates the motility and proliferation of FLSs and that the decrease in LERFS in RA FLSs might contribute to increased rheumatoid synovial aggression and hyperplasia, resulting in joint destruction. Indeed, consistent with our findings, a recent study also indicates that the lncRNA ZFAS1 is associated with RA FLS migration and invasion through miR-27a (46). In addition, a recent study has shown that RA FLSs in the sublining layer are different from those in the lining layer, in that they are not invasive (47); however, our data show that LERFS-positive cells were located close to the lining layer, which further supports our findings that LERFS is associated with an aggressive RA FLS phenotype.

We next explored the underlying molecular mechanism(s) by which LERFS regulates the migration and proliferation of RA FLSs. FISH and RT-qPCR of nuclear and cytoplasmic fractions revealed that LERFS is located in the cytoplasm, which suggests that it 

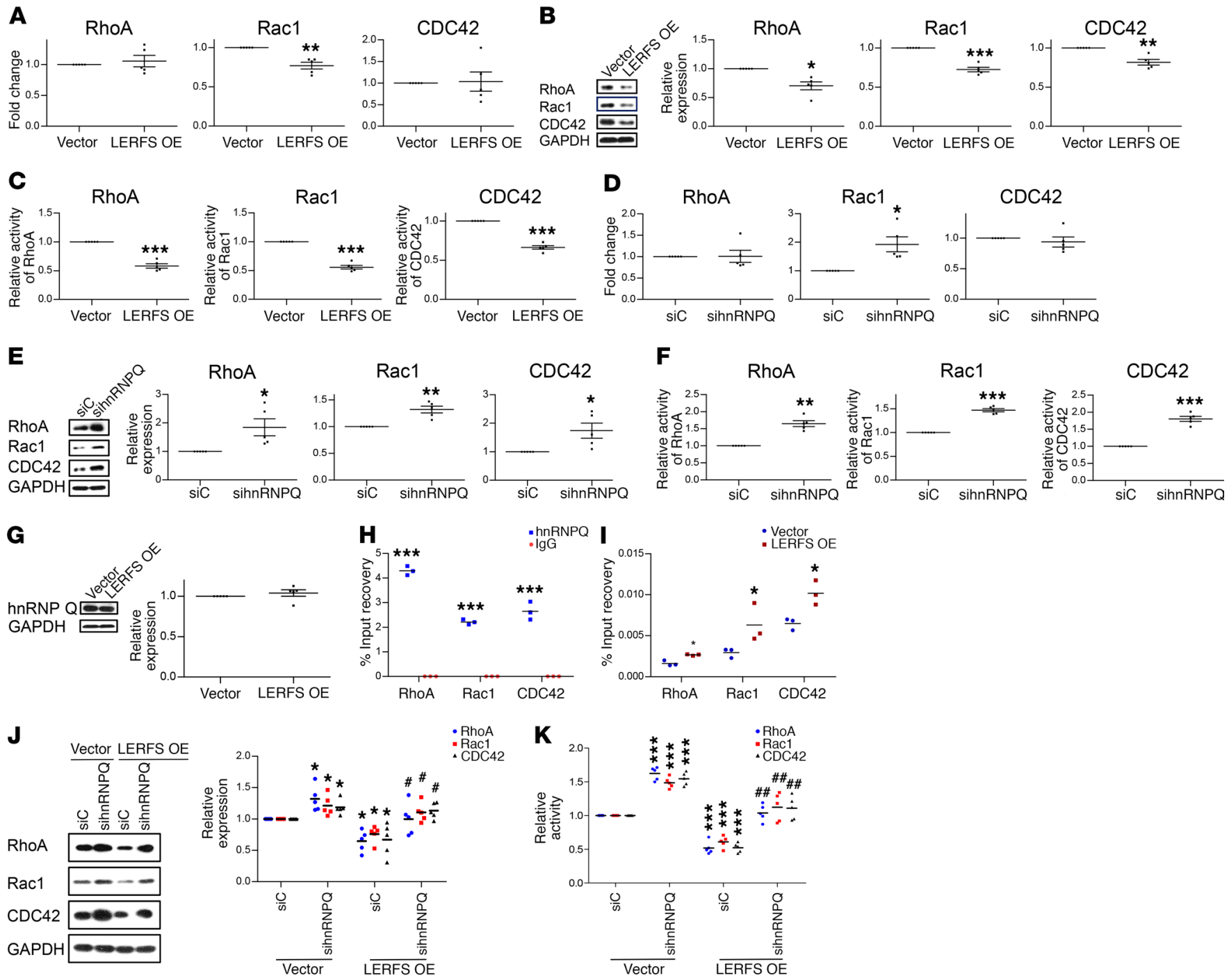

Figure 5. LERFS-hnRNP $\mathbf{Q}$ complex coregulates the expression of RhoA, Rac1, and CDC42. (A and B) Effect of LERFS overexpression on mRNA (A) and protein (B) expression of RhoA, Rac1, and CDC42 in RA FLSs. (C) Effect of LERFS overexpression on the activation of RhoA, Rac1, and CDC42. RhoA, Rac1, and CDC42 activity was measured by G-LISA. (D-F) Effect of hnRNP Q knockdown on expression levels of mRNA (D) and protein (E) and activity (F) of RhoA, Rac1, and CDC42. (G) Effect of LERFS overexpression on protein expression of hnRNP Q. (A-C) ${ }^{*} P<0.05,{ }^{*} P<0.01$, and ${ }^{* * *} P<0.001$ versus vector or siC, by Student's $t$ test. (H) RIP detection of the combination of hnRNP $Q$ and target mRNAs in RA FLSs. Values were normalized to the input. ${ }^{* *} P<0.001$ versus IgG, by Student's $t$ test. (I) Effect of LERFS overexpression on the association between hnRNP $Q$ and mRNA expression of RhoA, Rac1, and CDC42. Cell lysates from RA FLSs infected with control lentivirus (Vector) or LERFS OE were measured by RIP assay using antibodies against hnRNP Q or control IgG, followed by RT-qPCR assay of the indicated targets. Values were normalized to the input. ${ }^{*} P<0.05$ versus vector, by Student's $t$ test. (J and $\mathbf{K}$ ) Effect of hnRNP $\mathbf{k n n o c k d o w n}$ on LERFS overexpression-induced protein expression and activation of RhoA, Rac1, and CDC42. RA FLSs were transfected with hnRNP Q siRNA or siC for 24 hours, followed by infection of control lentivirus or LERFS OE. Three days later, cells were collected and subjected to Western blot analysis and G-LISA. Data shown are the quantification of protein levels $(\boldsymbol{J})$ and activity (K) of RhoA, Rac1, and CDC42. Data are expressed as the mean \pm SEM of 5 independent experiments involving 5 different RA patients. ${ }^{*} P<0.05$ and ${ }^{* * *} P<0.001$, versus siC plus vector; ${ }^{\#} P<0.05$ and ${ }^{\# \#} P<0.01$, versus siC plus LERFS OE, by 1 -way ANOVA.

might function by physically interacting with other cytoplasmic factors. Recent studies have shown a direct interaction between lncRNAs and cytoplasmic proteins $(21,34,48)$. In our study, LERFS was shown to function by forming a cytoplasmic RNAprotein complex with hnRNP Q, an RNA-binding protein involved in neuron motility $(34,38)$ but not previously documented to play a role in RA. In line with our findings, it has been reported that several lncRNAs function by binding with hnRNP members $(33,49)$. For instance, the lncRNA THRIL controls the expression of TNF- $\alpha$ in monocytes through its interaction with heterogeneous nuclear ribonucleoprotein L (hnRNPL) (33); lnc-HC binds to hnRNPA2B1 to modulate CYP7A1 and ABCA1 expression in hepatocytic cholesterol metabolism (49); and lnc13 regulates inflammatory gene expression by binding to heterogeneous nuclear ribonucleoprotein D (hnRNPD) in murine bone marrow-derived macrophages (50). However, our results do not completely rule out the possibility that other, unidentified cellular factors within the protein complex might mediate the interaction of LERFS with hnRNP Q.

The human hnRNP Q protein family consists of 3 isoforms: hnRNP Q1, Q2, and Q3. hnRNP Q1 is enriched in the cytoplasm, 


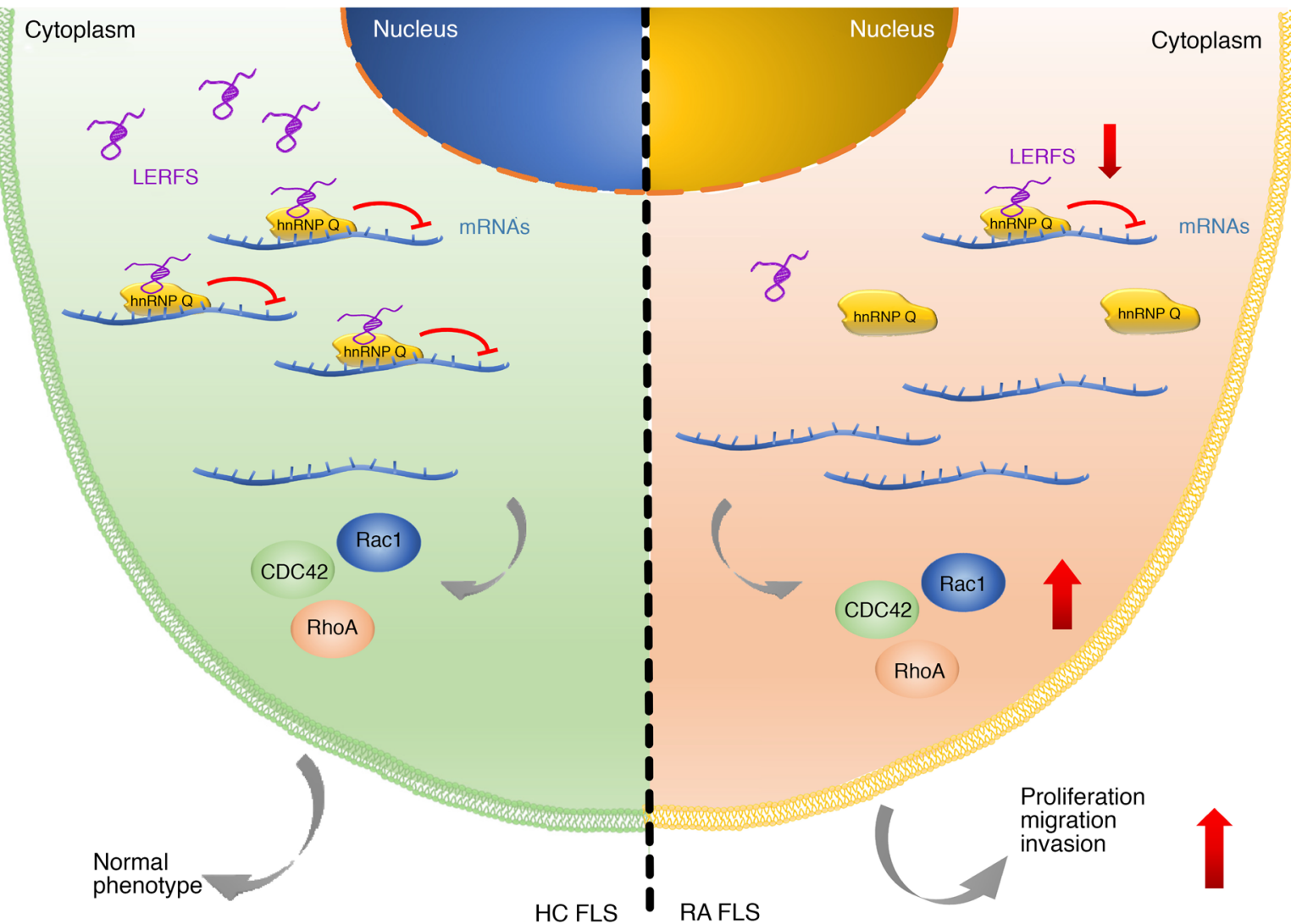

Figure 6. Proposed model for LERFS-mediated regulation of the migration, invasion, and proliferation of FLSs.

whereas hnRNP Q3 is located mostly in nuclei (36). These isoforms play important roles in RNA metabolism, such as cytoplasmic mRNA transport, pre-mRNA splicing, RNA editing, mRNA degradation, and translational activation (34-36, 51, 52). Previous reports have shown that hnRNP Q is involved in the localization of mRNAs that encode CDC42 signaling factors in neurites (38) as well as in the repression of mRNA translation and protein levels of RhoA (39). Moreover, Rho family proteins are involved in regulating the proliferation and aggressive behavior of RA FLSs. For instance, RhoA is considered a new target for the modulation of RA FLS invasion (41). Rac1 activation contributes to proliferation (42) and mediates IL-17A-induced migration of RA FLSs (53). This prompted us to propose that hnRNP Q functions through Rho proteins in RA FLSs. In the current study, we observed that hnRNP Q knockdown or overexpression, respectively, increased or reduced protein levels of RhoA, Rac1, and CDC42, but increased or reduced mRNA expression only in Rac1. These data suggest that hnRNP Q negatively modulates Rho protein levels through different regulatory mechanisms than those for mRNA metabolism. Further studies are needed to clarify the detailed mechanisms by which hnRNP Q modulates the mRNA metabolism of RhoA, Rac1, and CDC42 in FLSs.

We then addressed the question of whether hnRNP $Q$ is required for LERFS-mediated migration, invasion, and proliferation of RA FLSs. First, as with hnRNP Q, we observed that overexpression of LERFS suppressed protein levels of RhoA, Rac1, and CDC42 and affected mRNA levels of Rac1, but not of RhoA or CDC42. Interestingly, LERFS overexpression did not affect hnRNP Q expression. Second, RIP analysis revealed that hnRNP Q bound to the mRNAs of RhoA, Rac1, and CDC42 and that overexpression of LERFS could promote these combinations. Third, hnRNP Q knockdown could reverse the LERFS overexpressioninduced reduction of RhoA, Rac1, and CDC42 proteins. Moreover, our bioinformatics analysis also showed that LERFS could not bind directly to mRNA. Collectively, our findings suggest that a stable LERFS-hnRNP Q complex in the cytoplasm is a prerequisite for hnRNP Q binding to the mRNAs of RhoA, Rac1, and CDC42. In LERFS-decreased RA FLSs, formation of the LERFShnRNP Q complex may be prevented, which reduces the combination of hnRNP Q with RhoA, Rac1, and CDC42 mRNAs and promotes the stability or translation of these mRNAs and subsequent protein synthesis. In general, GTP-bound active RhoA, Rac1, and CDC42 levels are tightly controlled by their GAPs and GEFs (54). However, besides this conventional regulatory mechanism, recent reports indicate that the modulation of RhoA signaling activation can also be achieved by regulating Rho protein levels through different modes such as mRNA translation $(39,55)$, mRNA location (38), and specific protein degradation (54). Indeed, in our study, we also observed that LERFS overexpression suppressed the activation of RhoA, Rac1, and CDC42 in RA FLSs, which might have resulted from a LERFS overexpression-induced reduction of RhoA, Rac1, and CDC42 protein levels. Taken together, 
our findings provide evidence that LERFS bound specifically to hnRNP $Q$ and formed a functional LERFS-hnRNP Q complex that decreased the mRNA stability or translation of RhoA, Rac1, and CDC42 by binding to their mRNAs. However, we do not rule out the possibility that other unidentified factors might mediate the LERFS modulation of Rho GTPase activation.

In conclusion, we have identified the cytoplasmic lncRNA LERFS in FLSs and described its regulatory function in the migration, invasion, and proliferation of FLSs through interaction with hnRNP Q. Our study suggests that decreased expression of LERFS in FLSs might contribute to the synovial aggression and hyperplasia that characterize joint abnormalities in RA.

\section{Methods}

Preparation of human STs and FLSs. We obtained STs from active patients with RA (32 women and 2 men, 42-69 years of age) who were undergoing synovectomy of the knee joint or total knee replacement at the First Affiliated Hospital in Guangzhou, Guangdong, China. RA was diagnosed according to the 2010 American College of Rheumatology/ European League Against Rheumatism (ACR/EULAR) classification criteria (56). RA patients' demographics are provided in Supplemental Table 1. We obtained arthroscopic biopsies of HC STs from 29 subjects (27 women and 2 men, 39-68 years of age) who underwent arthroscopic surgery for knee meniscus injuries or cruciate ligament rupture and had no history of acute or chronic arthritis. We found no significant differences in sex or age between the RA patients and the HC subjects (Supplemental Table 2). We also obtained STs from 2 female patients with remitted RA. One patient underwent an arthroscopic biopsy for cruciate ligament rupture in the knee, and the other underwent a traumatic single, above-knee amputation. Both patients were treated with MTX and a TNF- $\alpha$ inhibitor (etanercept) before their operation.

STs were cut into small pieces and digested with $1 \mathrm{mg} / \mathrm{ml}$ collagenase for 3 hours at $37^{\circ} \mathrm{C}$ to isolate synoviocytes. All cells were cultured in DMEM/F12 with $10 \% \mathrm{FBS}$ at $5 \% \mathrm{CO}_{2}$ and $37^{\circ} \mathrm{C}$. In our experiments, we used cells from passages 4-6, during which time they were a homogeneous population of cells (1\% CD11b-positive, $1 \%$ phagocytic, and 1\% FcgRII- and FcgRIII receptor-positive). In addition, the human RA FLS cell line MH7A (Jennio Biotech Co. Ltd.), cultured as primary FLSs, was used in some experiments.

Microarray and data analysis. Total RNA was isolated with TRIzol (MilliporeSigma) from HC FLSs $(n=3)$ and RA FLSs $(n=3)$ and quantified using NanoDrop ND-1000 (Thermo Fisher Scientific). RNA integrity was assessed by standard denaturing agarose gel electrophoresis. After removal of rRNA using the mRNA-ONLY Eukaryotic mRNA Isolation Kit (Epicentre), the samples were amplified and transcribed into fluorescent cRNA along the entire length of the transcripts without 3 ' bias, using a random priming method. The labeled cRNAs were hybridized onto the Human LncRNA Array, version $2.0(8 \times 60 \mathrm{~K}$, Arraystar). After hybridization and washing, the arrays were scanned using the Agilent Scanner G2505B and Agilent Feature Extraction software, version 10.7.3.1, to analyze the acquired array images. Quantile normalization and subsequent data processing were performed using Agilent GeneSpring GX software, version 11.5.1. We identified differentially expressed lncRNAs with statistical significance through volcano plot filtering and calculated a P value using the Student's $t$ test. The threshold for up- and downregulated genes was a fold change of 2.0 or greater and a $P$ value of 0.05 or less. Heatmaps representing dif- ferentially regulated genes were generated using Cluster 3.0 software (ENCODE, Stanford University). Finally, we performed hierarchical clustering to show the distinguishable lncRNA expression patterns among the samples. The microarray data discussed in this article were deposited in the NCBI's Gene Expression Omnibus (GEO) database (GEO GSE103578; https://www.ncbi.nlm.nih.gov/geo/query/acc. cgi?acc $=$ GSE103578).

RACE. The SMARTer RACE cDNA Amplification Kit (Clontech) was used for rapid amplification of $5^{\prime}$ and $3^{\prime}$ ends of LERFS. The primer sequences for $5^{\prime}$ - and $3^{\prime}$-RACE are listed in Supplemental Table 3. RACE was conducted according to the manufacturer's instructions.

FISH. FISH was performed following the established protocol described previously (57). Cells were briefly rinsed in PBS and fixed in $4 \%$ formaldehyde in PBS (pH 7.4) for 15 minutes at room temperature (RT). Next, the cells were permeabilized in freshly made $0.5 \% \mathrm{v} / \mathrm{v}$ Triton X-100 in PBS containing $2 \mathrm{mM}$ voriconazole (VRC) on ice for 10 minutes. After rinsing them with $2 \times$ SSC, the cells were hybridized with double-digoxigenin-labeled (DIG-labeled) locked nucleic acid (LNA) detection probes ( $80 \mathrm{nM}$; Exiqon, Vedbaek) at $37^{\circ} \mathrm{C}$ for 16 hours in a moist chamber. After stringent washes, the cells were incubated at RT for 1 hour with an anti-DIG-fluorescein monoclonal antibody (1:200; catalog 11207741910, Roche Life Science). Cells were counterstained with DAPI and imaged using a confocal laser scanning microscope (LSM 710, Carl Zeiss) equipped with LSM ZEN 2008 software.

$I S H$. To detect the expression pattern and location of LERFS in STs, ISH was performed with DIG-labeled LNA detection probes (Exiqon). Briefly, after deparaffinization and rehydration, the samples were digested with proteinase $\mathrm{K}(15 \mu \mathrm{g} / \mathrm{ml})$ for 10 minutes at $37^{\circ} \mathrm{C}$ and subsequently dehydrated. The sections were then hybridized with a probe $(80 \mathrm{nM})$ at $54^{\circ} \mathrm{C}$ for 1 hour. After stringent washes and blocking, the sections were incubated at RT for 1 hour with an anti-DIGalkaline phosphatase (AP) monoclonal antibody (1:800; catalog 11093274910, Roche Life Science). Then, the sections were stained with nitroblue tetrazolium/5-bromo-4-chloro-3-indolyl phosphate (NBT/BCIP) (Roche Life Science), counterstained with nuclear fast red, and mounted.

In vitro transcription and translation. We used the TNT Quick Coupled Transcription/Translation System (Promega) to detect the protein-coding capacity of the lncRNA, conducting in vitro transcription and translation according to the manufacturer's instructions. We cloned the lncRNA into pcDNA3.1 downstream of the T7 promoter, translated it in vitro, and stained it with the FluoroTect Green Lys In Vitro Translation Labeling System (Promega). Luciferase mRNA was used as a positive translation control and a mock-translated sample (no RNA template) as a NC. The fluorescent translation product was resolved by SDS-PAGE and then visualized using a Typhoon 8600 Phosphorimager (GE Healthcare Life Sciences) with $532 \mathrm{~nm}$ excitation.

RNA-protein-pulldown assay. Full-length sense and antisense LERFS were amplified by PCR and cloned using a pGEM-T Vector System (Promega). Biotin-labeled RNAs were generated by an in vitro transcription reaction with Biotin RNA Labeling Mix and T7 RNA polymerase (both from Roche Life Science) and then treated with RNaseFree DNase I (Promega). Biotinylated RNAs $(1 \mu \mathrm{g})$ were refolded into RNA structure buffer (10 mM Tris pH 7, $0.1 \mathrm{M} \mathrm{KCl,} 10 \mathrm{mM} \mathrm{MgCl}$ ) at $95^{\circ} \mathrm{C}$ for 2 minutes, cooled on ice for 3 minutes, and then left at RT for 30 minutes to form a proper secondary structure. Streptavidin beads were washed on ice and mixed with the folded RNA, left overnight 
at $4^{\circ} \mathrm{C}$, and then collected and washed. Total cell lysates (containing $1 \mathrm{mg}$ proteins) were incubated with the RNA-bead mixture at RT for 1 hour. The beads were then collected and washed 3 times. RNAbinding protein complexes were eluted and denatured with SDS loading buffer and resolved by SDS-PAGE followed by silver staining. Finally, MS using a Linear Ion Trap Mass Spectrometer (Thermo Fisher Scientific) and Western blot assays to identify proteins associated with LERFS were performed.

RIP. For RIP, cells were lysed with cell lysis buffer (Cell Signaling Technology) supplemented with PMSF (Genstar). Protein concentrations were measured by BCA protein assay (Thermo Fisher Scientific). RIP was performed using the RNA-Binding Protein Immunoprecipitation Kit (MilliporeSigma) according to the manufacturer's instructions. Magnetic beads $(50 \mu \mathrm{l})$ were washed twice, collected, and then resuspended in $100 \mu \mathrm{l}$ RIP wash buffer. hnRNP Q antibody $(5 \mu \mathrm{g}$, cata$\log$ ab10687, Abcam) was added to the tube and incubated for $30 \mathrm{~min}$ utes at RT. The beads-antibody complex was washed, mixed with 100 $\mu \mathrm{l}$ cell lysates in $900 \mu \mathrm{l}$ RIP buffer, and the samples incubated at $4^{\circ} \mathrm{C}$ overnight. Immunoprecipitated products were washed, collected, and then treated with proteinase K. Finally, total RNA was extracted from the immunoprecipitated samples and subjected to RT-qPCR analysis.

$R T-q P C R$. Total RNA was prepared from FLSs using the Takara PrimeScript RT Reagent Kit (Takara Bio) according to the manufacturer's protocol, and RT-qPCR was performed using the Bio-Rad CFX96 system. The primers used for real-time PCR are listed in Supplemental Table 3. To quantify the relative expression of each gene, Ct values were normalized to the endogenous reference $(\Delta \mathrm{Ct}=\mathrm{Ct}$ target $-\mathrm{Ct}$ GAPDH $)$ and compared using a calibrator and the $\Delta \Delta \mathrm{Ct}$ method $(\Delta \Delta \mathrm{Ct}=\Delta \mathrm{Ct}$ sample $-\Delta$ Ct calibrator). All experiments were performed in triplicate.

Western blot analysis. Protein concentrations were detected using the Bicinchoninic Acid Protein Assay Kit (Thermo Fisher Scientific). Equal amounts of protein were solubilized in Laemmli buffer $(62.5 \mathrm{mM}$ Tris-HCl, pH 6.8, 10\% glycerol, $2 \%$ SDS, $5 \%$-mercaptoethanol, and $0.00625 \%$ bromophenol blue), boiled for 5 minutes, separated by SDSPAGE, and transferred onto nitrocellulose membranes. The membranes were probed with the following primary antibodies: anti-hnRNP Q (ab10687, Abcam), anti-RhoA (2117, Cell Signaling Technology), anti-CDC42 (ab64533, Abcam), anti-Rac1 (ab33186, Abcam), and anti-GAPDH (G8795, MilliporeSigma) in TBS/Tween-20 containing $5 \%$ nonfat milk at $4^{\circ} \mathrm{C}$ overnight. The membranes were incubated with the appropriate secondary antibodies for 1 hour at RT. Immunoreactive bands were visualized using ECL (GE Healthcare). Each blot is representative of at least 3 similar independent experiments.

Infection of overexpression lentivirus. We purchased LERFS- and hnRNP Q-overexpressed lentiviruses were purchased from Genechem; empty vector lentiviruses expressing GFP were used as NCs only. RA FLSs were cultured in 6-well plates until 60\% confluent and infected with lentivirus particles in the presence of $10 \mathrm{~g} / \mathrm{ml}$ polybrene at a MOI of 30 . The cells were cultured for at least 3 days before further experiments were performed.

Transfection of LncRNA Smart Silencer and siRNA. We used LncRNA Smart Silencer, synthesized by RiboBio, to target LERFS. LERFS Smart Silencer was a mixture of 3 siRNAs and 3 ASOs. The NC Smart Silencer did not contain domains homologous to those in humans, mice, or rats. hnRNP Q siRNAs and nonsilencing control siRNAs were obtained from RiboBio as well. The target sequences of siRNAs are listed in Supplemental Table 3. Cells were seeded in 6-well plates at
$60 \%$ to $70 \%$ confluence and transiently transfected with hnRNP Q siRNA (50 nM), LERFS Smart Silencer (100 nM), or a corresponding NC using Lipofectamine RNAiMAX Reagent (Thermo Fisher Scientific). Experiments were performed 48 hours after transfection.

Measurement of cell migration and invasion in vitro. A chemotaxis assay of FLSs was performed with the Boyden chamber method using a 6.5-mm filter with a pore size of $8.0 \mu \mathrm{m}$ (Transwell, Corning Labware Products). Briefly, DMEM containing 10\% FBS or MCP-1 (50 ng/ml, R\&D Systems) was placed as a chemoattractant in the lower wells, and FLSs were suspended at a final concentration of $6 \times 10^{4} \mathrm{cells} / \mathrm{ml}$ in serumfree DMEM in the upper wells. The plate was incubated at $37^{\circ} \mathrm{C}$ in $5 \%$ $\mathrm{CO}_{2}$ for 12 hours. After incubation, the nonmigrating cells were removed from the filter's upper surface using a cotton swab. The filters were fixed in methanol for 15 minutes and stained with $0.1 \%$ crystal violet for $15 \mathrm{~min}$ utes. Chemotaxis was quantified using an optical microscope to count the stained cells that had migrated to the lower side of the filter. The stained cells were counted as the mean number of cells per 10 random fields for each assay. For the in vitro invasion assay, similar experiments were conducted using inserts coated with BD Matrigel Basement Membrane Matrix (BD Biosciences) and DMEM containing 10\% FBS as a chemoattractant. The plate was incubated at $37^{\circ} \mathrm{C}$ in $5 \% \mathrm{CO}_{2}$ for 48 hours.

Wounding migration. RA FLSs, plated to $70 \%$ confluence on $35-\mathrm{mm}$ culture dishes, were serum starved for 12 to 16 hours and wounded with 200- $\mu$ l pipette tips. The culture dishes were washed 3 times with PBS to remove detached cells, and the remaining cells were grown in DMEM containing $10 \%$ FBS. After 48 hours of incubation, migration was quantified by counting the cells that had moved beyond a reference line.

Determination of migration of FLSs in vivo. We adopted a recently reported in vivo assay to examine whether overexpression of LERFS inhibits the in vivo migration of RA FLSs $(58,59)$. Eight-week-old male BALB/c athymic nude mice were obtained from the Guangdong Medical Laboratory Animal Center (Guangzhou, China). All mice were fed normal mouse chow and water ad libitum and housed under standard conditions with air filtration $\left(20^{\circ} \mathrm{C} \pm 2{ }^{\circ} \mathrm{C} ; 12-\mathrm{h}\right.$ light/12-h dark cycle). RA FLSs were infected with control lentivirus (vector) or LERFS-overexpressing lentivirus (LERFS OE) for 5 days. CFA $(120 \mu \mathrm{g})$ was subcutaneously injected into athymic nude mice to induce skin inflammation. After 1 day, RA FLSs $\left(5 \times 10^{5}\right)$ were injected intradermally into the backs of these mice, $1.2 \mathrm{~cm}$ from the CFA injection site. After 5 days, skin samples were obtained from the FLSimplanted site and CFA-injected site, as well as from areas between the 2 sites. Immunohistochemical staining was performed on the samples using anti-human class I HLA antibody (1:50, ab70328, Abcam) to detect migrated and invading FLSs. We then observed and counted the number of HLA class I-positive cells, indicating human RA FLSs, under light microscopy.

Determination of in vivo invasion of RA FLSs into human cartilage implants. Before implantation, cultured FLSs were trypsinized and resuspended in sterile saline solution to a final volume of $60 \mathrm{ml}$ for each sponge. The normal human cartilage, obtained from nonarthritic patients undergoing knee surgery for traumatic injuries, was cut into 5 - to $8-\mathrm{mm}^{3}$ pieces. One piece, as a control for scoring after implantation, was immediately snap-frozen and stored at $-70^{\circ} \mathrm{C}$. For implantation, 2 implants containing cartilage, and the same population of RA FLSs were inserted under the skin of the left flanks of 4-week-old SCID mice (Guangdong Medical Laboratory Animal Center, Guangzhou, China). In brief, on the day of implantation, normal human cartilage 
was obtained and cut into pieces. A cube of insert sponge $\left(80 \mathrm{~mm}^{3}\right)$ was incised, and a piece of cartilage $\left(5-8 \mathrm{~mm}^{3}\right)$ was inserted. The sponge was soaked with $4 \times 10^{5} \mathrm{FLSs}$ suspended in sterile saline. Three pieces of sponge containing FLSs and cartilage were inserted into the skin of the anesthetized mice under sterile conditions. After 50 days, the implants were removed from the sacrificed mice and immediately embedded, snap-frozen, and stored at $-70^{\circ} \mathrm{C}$ until further use. The explants were stained with standard $\mathrm{H} \& \mathrm{E}$, and each specimen was examined for the grade of FLS invasion into cartilage as described previously (60). The level of invasiveness was scored as follows: $0=$ no or minimal invasion; 1 = visible invasion (2-cell depth); 2 = invasion (5-cell depth); and 3 = deep invasion (more than 10-cell depth). The sections were examined in a blinded manner by 2 experienced investigators.

$E d U$ proliferation assays. Cells were cultured in 96-well plates in complete media until $80 \%$ to $90 \%$ confluent and then incubated with $50 \mu \mathrm{M}$ 5-ethynyl-2'-deoxyuridine (EdU) in complete media for 6 hours. The Cell-Light EdU DNA Cell Proliferation Kit (RiboBio) was used to measure FLS proliferation according to the manufacturer's instructions.

MTT assay. Cells $\left(3 \times 10^{3}\right.$ cells/well $)$ were seeded into 96-well plates and incubated for 24 hours. After lentivirus infection, the cells were incubated with a solution of MTT salt $(5 \mathrm{mg} / \mathrm{ml}$ in PBS) for 30 minutes at $37^{\circ} \mathrm{C}$, and then the dark blue crystals of formazan produced in acidified isopropanol were dissolved and the amount of formazan quantified by reading the OD at $490 \mathrm{~nm}$.

Cell-cycle analysis. The effect of LERFS overexpression on the cell cycle was detected by flow cytometry. Briefly, $1 \times 10^{5}$ cells per well were seeded into 6-well plates and incubated for 24 hours. The cells were then harvested and washed with PBS. Next, the pellet was resuspended, fixed in $70 \%$ prechilled methanol, and stored overnight at $-20^{\circ} \mathrm{C}$. The cells were washed again with PBS followed by addition of $200 \mu \mathrm{l}$ staining solution ( $0.1 \%$ [v/v] Triton X-100, $1 \mu \mathrm{g} / \mathrm{ml}$ DAPI in PBS) into the pellet. The final mixture was incubated for 30 minutes in darkness before flow cytometric analysis. The experiments were performed in triplicate and repeated 3 times.

Apoptosis assays. FLS apoptosis was analyzed by staining cells with allophycocyanin (APC) annexin V and 7-aminoactinomycin D (7-AAD) (both from BD Biosciences) according to the manufacturer's protocol. Briefly, cells were suspended in $1 \times$ binding buffer at a concentration of $1 \times 10^{6}$ cells $/ \mathrm{ml}$. The cell suspension $(100 \mu \mathrm{l})$ was then transferred to a 1.5-ml Eppendorf tube, mixed with $5 \mu \mathrm{l}$ APC annexin $\mathrm{V}$ and $10 \mu \mathrm{l}$ 7-AAD, and incubated for 20 minutes at RT in darkness. Within 1 hour, the samples were analyzed by flow cytometry.

Detection of RhoA, Racl, and CDC42 activity. Cells were cultured for 24 hours at a density of $1 \times 10^{5}$ cells/well in $35-\mathrm{mm}$ culture dishes in serum-free medium. The activity of RhoA, Rac1, and CDC42 was measured using a G-LISA RhoA, Rac1, and CDC42 Activation Assay Kit (Cytoskeleton) according to the manufacturer's instructions.
Statistics. Data are expressed as the mean \pm SEM. The data presented were derived from at least 5 independent experiments for the in vitro experiments. The experimental procedures and treatment and data analyses were performed in a blinded manner. To reduce baseline variability between independent experiments, the quantitative analysis of immunoblots and mRNA expression was normalized. Data were normalized to the fold change over the mean of the control. Twogroup comparisons were made using a 2-tailed Student's $t$ test; 3 or more different groups were evaluated by 1-way ANOVA followed by Bonferroni's post hoc comparisons. A $P$ value of less than 0.05 was considered significant. Statistical analyses of the data were performed using SPSS, version 13.0 (IBM).

Study approval. All studies were performed according to Declaration of Helsinki principles and approved by the Medical Ethics Committee of the First Affiliated Hospital of Sun Yat-sen University. All patients provided written informed consent to participate in the study. Animal handling and procedures were approved by the Animal Care and Ethics Committee of Sun Yat-sen University.

\section{Author contributions}

YZ, SX, and YX performed the majority of the experiments and analyzed and interpreted the data. QQ, JW, ZZ, and LL collected clinical samples and analyzed and interpreted the data. MS performed the in vivo experiments. XY, SZ, and HX contributed to the study concept and design. YZ, NO, SZ, and HX drafted the manuscript. SZ and HX contributed to the supervision of the study.

\section{Acknowledgments}

The authors would like to thank Nunzio Bottini (UCSD, San Diego, California, USA) for his critical comments on the manuscript. This work was supported by grants from the National Natural Science Foundation of China (81671591, U1401222, 81671611, 81501389, and 81373182); the Fundamental Research Funds for the Central Universities (17ykjc07); the Guangdong Natural Science Foundation (S2011020002358 and S2013010015363); the Guangdong Science and Technology Project (2016A020215051); the Zhujiang Innovative and Entrepreneurial Talent Team Award of Guangdong Province (2016 ZT 06S 252); and the NIH (R01 AR059103).

Address correspondence to: Hanshi Xu, Department of Rheumatology, First Affiliated Hospital, Sun Yat-sen University, 58 Zhongshan Road 2, Guangzhou, Guangdong 510080, China. Phone: 8620.87755766; Email: xuhanshi@mail.sysu.edu.cn. Or to: Song Guo Zheng, Penn State Hershey Medical Center, 500 University Drive, Hershey, Pennsylvania 17033, USA. Phone: 717.531.0003; Email: szheng1@pennstatehealdh.psu.edu.
1. Huber LC, Distler O, Tarner I, Gay RE, Gay S, Pap T. Synovial fibroblasts: key players in rheumatoid arthritis. Rheumatology (Oxford). 2006;45(6):669-675.

2. Müller-Ladner U, Pap T, Gay RE, Neidhart M, Gay S. Mechanisms of disease: the molecular and cellular basis of joint destruction in rheumatoid arthritis. Nat Clin Pract Rheumatol. 2005;1(2):102-110.

3. Lefevre S, Meier FM, Neumann E, Muller-Ladner
U. Role of synovial fibroblasts in rheumatoid arthritis. Curr Pharm Des. 2015;21(2):130-141.

4. Bottini N, Firestein GS. Duality of fibroblast-like synoviocytes in RA: passive responders and imprinted aggressors. Nat Rev Rheumatol. 2013;9(1):24-33.

5. Lefèvre $\mathrm{S}$, et al. Synovial fibroblasts spread rheumatoid arthritis to unaffected joints. Nat Med. 2009;15(12):1414-1420.

6. Roivainen A, Jalava J, Pirilä L, Yli-Jama T,
Tiusanen H, Toivanen P. H-ras oncogene point mutations in arthritic synovium. Arthritis Rheum. 1997;40(9):1636-1643.

7. Firestein GS, Echeverri F, Yeo M, Zvaifler NJ, Green DR. Somatic mutations in the p53 tumor suppressor gene in rheumatoid arthritis synovium. Proc Natl Acad Sci U S A. 1997;94(20):10895-10900.

8. Frank-Bertoncelj M, et al. Epigenetically-driven anatomical diversity of synovial fibroblasts 
guides joint-specific fibroblast functions. Nat Commun. 2017;8:14852

9. Ntougkos E, et al. Genomic Responses of Mouse Synovial Fibroblasts During Tumor Necrosis Factor-Driven Arthritogenesis Greatly Mimic Those in Human Rheumatoid Arthritis. Arthritis Rheumatol. 2017;69(8):1588-1600.

10. Djebali S, et al. Landscape of transcription in human cells. Nature. 2012;489(7414):101-108.

11. Harrow J, et al. GENCODE: the reference human genome annotation for The ENCODE Project. Genome Res. 2012;22(9):1760-1774.

12. Elgar G, Vavouri T. Tuning in to the signals: noncoding sequence conservation in vertebrate genomes. Trends Genet. 2008;24(7):344-352.

13. Nakamachi Y, et al. MicroRNA-124a is a key regulator of proliferation and monocyte chemoattractant protein 1 secretion in fibroblast-like synoviocytes from patients with rheumatoid arthritis. Arthritis Rheum. 2009;60(5):1294-1304.

14. Trenkmann M, Brock M, Gay RE, Michel BA, Gay S, Huber LC. Tumor necrosis factor $\alpha$-induced microRNA-18a activates rheumatoid arthritis synovial fibroblasts through a feedback loop in NF-кB signaling. Arthritis Rheum. 2013;65(4):916-927.

15. Lin J, et al. A novel p53/microRNA-22/Cyr61 axis in synovial cells regulates inflammation in rheumatoid arthritis. Arthritis Rheumatol. 2014;66(1):49-59.

16. Guttman $\mathrm{M}$, et al. Chromatin signature reveals over a thousand highly conserved large non-coding RNAs in mammals. Nature. 2009;458(7235):223-227.

17. Huarte $\mathrm{M}$, et al. A large intergenic noncoding RNA induced by p53 mediates global gene repression in the $\mathrm{p} 53$ response. Cell. 2010;142(3):409-419.

18. Divoux A, et al. Identification of a novel lncRNA in gluteal adipose tissue and evidence for its positive effect on preadipocyte differentiation. Obesity (Silver Spring). 2014;22(8):1781-1785.

19. Hung T, et al. Extensive and coordinated transcription of noncoding RNAs within cell-cycle promoters. Nat Genet. 2011;43(7):621-629.

20. Li P, et al. A liver-enriched long non-coding RNA, lncLSTR, regulates systemic lipid metabolism in mice. Cell Metab. 2015;21(3):455-467.

21. Wang P, et al. The STAT3-binding long noncoding RNA lnc-DC controls human dendritic cell differentiation. Science. 2014;344(6181):310-313.

22. Tsai MC, et al. Long noncoding RNA as modular scaffold of histone modification complexes. Science. 2010;329(5992):689-693.

23. Guttman M, et al. lincRNAs act in the circuitry controlling pluripotency and differentiation. Nature. 2011;477(7364):295-300.

24. Wang KC, et al. A long noncoding RNA maintains active chromatin to coordinate homeotic gene expression. Nature. 2011;472(7341):120-124.

25. Ulitsky I, Shkumatava A, Jan CH, Sive H, Bartel DP. Conserved function of lincRNAs in vertebrate embryonic development despite rapid sequence evolution. Cell. 2011;147(7):1537-1550.

26. Prensner JR, et al. Transcriptome sequencing across a prostate cancer cohort identifies PCAT-1, an unannotated lincRNA implicated in disease progression. Nat Biotechnol. 2011;29(8):742-749.

27. Gupta RA, et al. Long non-coding RNA HOTAIR reprograms chromatin state to promote cancer metastasis. Nature. 2010;464(7291):1071-1076.

28. Deng M, Blondeau JJ, Schmidt D, Perner S, Müller SC, Ellinger J. Identification of novel differentially expressed lncRNA and mRNA transcripts in clear cell renal cell carcinoma by expression profiling. Genom Data. 2015;5:173-175.

29. Chen Z, et al. cAMP/CREB-regulated LINC00473 marks LKB1-inactivated lung cancer and mediates tumor growth. J Clin Invest. 2016;126(6):2267-2279.

30. Morán I, et al. Human $\beta$ cell transcriptome analysis uncovers lncRNAs that are tissue-specific, dynamically regulated, and abnormally expressed in type 2 diabetes. Cell Metab. 2012;16(4):435-448.

31. Archer K, et al. Long Non-Coding RNAs as Master Regulators in Cardiovascular Diseases. Int J Mol Sci. 2015;16(10):23651-23667.

32. Wu Y, et al. Association of large intergenic noncoding RNA expression with disease activity and organ damage in systemic lupus erythematosus. Arthritis Res Ther. 2015;17:131.

33. Li Z, et al. The long noncoding RNA THRIL regulates TNF $\alpha$ expression through its interaction with hnRNPL. Proc Natl Acad Sci US A. 2014;111(3):1002-1007.

34. Bannai H, et al. An RNA-interacting protein, SYNCRIP (heterogeneous nuclear ribonuclear protein Q1/NSAP1) is a component of mRNA granule transported with inositol 1,4,5-trisphosphate receptor type $1 \mathrm{mRNA}$ in neuronal dendrites. J Biol Chem. 2004;279(51):53427-53434.

35. Blanc V, et al. Identification of GRY-RBP as an apolipoprotein B RNA-binding protein that interacts with both apobec- 1 and apobec- 1 complementation factor to modulate $\mathrm{C}$ to $\mathrm{U}$ editing. J Biol Chem. 2001;276(13):10272-10283.

36. Chen HH, Chang JG, Lu RM, Peng TY, Tarn WY. The RNA binding protein hnRNP Q modulates the utilization of exon 7 in the survival motor neuron 2 (SMN2) gene. Mol Cell Biol. 2008;28(22):6929-6938.

37. Kim DY, Woo KC, Lee KH, Kim TD, Kim KT. hnRNP $Q$ and PTB modulate the circadian oscillation of mouse Rev-erb alpha via IRES-mediated translation. Nucleic Acids Res. 2010;38(20):7068-7078.

38. Chen HH, Yu HI, Chiang WC, Lin YD, Shia BC, Tarn WY. hnRNP Q regulates Cdc42mediated neuronal morphogenesis. Mol Cell Biol. 2012;32(12):2224-2238.

39. Xing L, Yao X, Williams KR, Bassell GJ. Negative regulation of RhoA translation and signaling by hnRNP-Q1 affects cellular morphogenesis. $\mathrm{Mol}$ Biol Cell. 2012;23(8):1500-1509.

40. Remmers EF, Sano H, Wilder RL. Plateletderived growth factors and heparin-binding (fibroblast) growth factors in the synovial tissue pathology of rheumatoid arthritis. Semin Arthritis Rheum.1991;21(3):191-199.

41. Xiao Y, et al. Inhibitory effects of simvastatin on migration and invasion of rheumatoid fibroblast-like synoviocytes by preventing geranylgeranylation of RhoA. Rheumatol Int. 2013;33(2):389-399.
42. Chan A, Akhtar M, Brenner M, Zheng Y, Gulko PS, Symons M. The GTPase Rac regulates the proliferation and invasion of fibroblast-like synoviocytes from rheumatoid arthritis patients. Mol Med. 2007;13(5-6):297-304.

43. McGarry T, Veale DJ, Gao W, Orr C, Fearon U, Connolly M. Toll-like receptor 2 (TLR2) induces migration and invasive mechanisms in rheumatoid arthritis. Arthritis Res Ther. 2015;17:153.

44. Zhang Y, et al. Long noncoding RNA expression profile in fibroblast-like synoviocytes from patients with rheumatoid arthritis. Arthritis Res Ther. 2016;18(1):227.

45. Mo BY, et al. Long Non-Coding RNA GAPLINC promotes tumor-like biologic behaviors of fibroblast-like synoviocytes as microRNA sponging in rheumatoid arthritis patients. Front Immunol. 2018;9:702.

46. Ye Y, Gao X, Yang N. LncRNA ZFAS1 promotes cell migration and invasion of fibroblast-like synoviocytes by suppression of miR-27a in rheumatoid arthritis. Hum Cell. 2018;31(1):14-21.

47. Croft AP, et al. Rheumatoid synovial fibroblasts differentiate into distinct subsets in the presence of cytokines and cartilage. Arthritis Res Ther. 2016;18(1):270.

48. Liu B, et al. A cytoplasmic NF- $\kappa \mathrm{B}$ interacting long noncoding RNA blocks IкB phosphorylation and suppresses breast cancer metastasis. Cancer Cell. 2015;27(3):370-381.

49. Lan X, et al. A novel long noncoding RNA Lnc-HC binds hnRNPA2B1 to regulate expressions of Cyp7a1 and Abca1 in hepatocytic cholesterol metabolism. Hepatology. 2016;64(1):58-72.

50. Castellanos-Rubio A, et al. A long noncoding RNA associated with susceptibility to celiac disease. Science. 2016;352(6281):91-95.

51. Grosset C, Chen CY, Xu N, Sonenberg N, Jacquemin-Sablon H, Shyu AB. A mechanism for translationally coupled mRNA turnover: interaction between the poly(A) tail and a c-fos RNA coding determinant via a protein complex. Cell. 2000;103(1):29-40

52. Kanai Y, Dohmae N, Hirokawa N. Kinesin transports RNA: isolation and characterization of an RNA-transporting granule. Neuron. 2004;43(4):513-525.

53. Moran EM, Connolly M, Gao W, McCormick J, Fearon U, Veale DJ. Interleukin-17A induction of angiogenesis, cell migration, and cytoskeletal rearrangement. Arthritis Rheum. 2011;63(11):3263-3273.

54. Sit ST, Manser E. Rho GTPases and their role in organizing the actin cytoskeleton. J Cell Sci. 2011;124(Pt 5):679-683.

55. Chiba Y, Tanabe M, Goto K, Sakai H, Misawa M. Down-regulation of miR-133a contributes to up-regulation of Rhoa in bronchial smooth muscle cells. Am J Respir Crit Care Med. 2009;180(8):713-719.

56. Aletaha D, et al. 2010 Rheumatoid arthritis classification criteria: an American College of Rheumatology/European League Against Rheumatism collaborative initiative. Arthritis Rheum. 2010;62(9):2569-2581.

57. Tripathi V, Fei J, Ha T, Prasanth KV. RNA fluorescence in situ hybridization in cultured mammalian cells. Methods Mol Biol. 2015;1206:123-136. 
58. Stanford SM, et al. TGF $\beta$ responsive tyrosine phosphatase promotes rheumatoid synovial fibroblast invasiveness. Ann Rheum Dis. 2016;75(1):295-302.
59. You S, et al. Identification of key regulators for the migration and invasion of rheumatoid synoviocytes through a systems approach. Proc Natl Acad Sci U S A. 2014;111(1):550-555.
60. Müller-Ladner U, et al. Gene transfer of cytokine inhibitors into human synovial fibroblasts in the SCID mouse model. Arthritis Rheum. 1999;42(3):490-497. 